\title{
TRANSFORMING STRUCTURES BY SET INTERPRETATIONS
}

\author{
THOMAS COLCOMBET ${ }^{a}$ AND CHRISTOF LÖDING ${ }^{b}$ \\ ${ }^{a}$ Cnrs-Irisa, Irisa, Campus de Beaulieu, 35042 Rennes, France \\ e-mail address: Thomas.Colcombet@irisa.fr \\ ${ }^{b}$ RWTH Aachen, Informatik 7, Ahornstr. 55, Aachen, Germany \\ e-mail address: loeding@informatik.rwth-aachen.de
}

\begin{abstract}
We consider a new kind of interpretation over relational structures: finite sets interpretations. Those interpretations are defined by weak monadic second-order (WMSO) formulas with free set variables. They transform a given structure into a structure with a domain consisting of finite sets of elements of the orignal structure. The definition of these interpretations directly implies that they send structures with a decidable WMSO theory to structures with a decidable first-order theory. In this paper, we investigate the expressive power of such interpretations applied to infinite deterministic trees. The results can be used in the study of automatic and tree-automatic structures.
\end{abstract}

\section{INTRODUCTION}

Computational model theory is concerned with the study of algorithmic properties of classes of infinite structures (cf. [BG04]), where the focus is on the problem of model checking such structures against specifications written in some logic, i.e., deciding for a given structure and logical formula if the formula holds in this structure. This problem setting has been studied for various instantiations of the two parameters, i.e., the way to represent the structures, and the logic to write the specifications. The most prominent logics in this context are first-order (FO) logic and monadic second-order (MSO) logic, and they have led to two tracks of research trying to identify classes of structures for which the respective logic is decidable.

One way of defining such classes uses, e.g., words or trees for representing the elements of the structure and uses simple transformations based on transducers or rewriting to define the relations of the structure. In this way, one obtains, e.g., the classes of automatic [Hod83, KN95, BG00] and tree-automatic [DT90, BG00] structures, for which the FOtheory is decidable, and the classes of pushdown-graphs [MS85 and prefix recognizable graphs Cau96 with decidable MSO-theory.

2000 ACM Subject Classification: F.4.1.

Key words and phrases: infinite structures, decidable theories, monadic second-order logic, first-order logic, automatic structures. 
In the case of automatic structures, the decidability results are based on the strong closure properties of finite automata, which are used to define the relations. Other techniques, e.g. in [KL02 for rewriting in trace monoids, are based on Gaifman's locality theorem.

The decidability results for MSO logic on pushdown and prefix recognizable graphs are derived from the results of Büchi and Rabin establishing the equivalence of monadic secondorder logic with certain families of finite automata accepting infinite trees (cf. [Tho97]). These results are also underlying the more recent work [CT02] and KNUW05] showing the decidability of MSO logic over certain classes of infinite words and infinite trees, respectively.

A different and more systematic approach for defining and studying classes of infinite structures is to use operations for transforming structures. An important operation of this kind is the model-theoretic interpretation. Such an interpretation defines a new structure 'inside' a given one by means of logical formulas describing the domain and the new relations. Depending on whether these defining formulas are FO or MSO one speaks of FO- and MSO-interpretations. An important property of these interpretations is that decidability results easily transfer from the given structure to the resulting structure, i.e., applying an FO-interpretation to a structure with a decidable FO-theory results in a structure with decidable FO-theory, and similarly for MSO.

As mentioned in BG04, this suggests a new way of defining interesting classes of infinite structures: fix an underlying structure (with good algorithmic properties) and consider all structures that can be obtained by applying interpretations of a certain kind. In this way, one obtains the automatic structures by FO-interpretations from, e.g., a suitable extension of Presburger arithmetic [Blu99], and the prefix recognizable structures by MSOinterpretations from the infinite binary tree Blu01. This idea has been pursued further in Cau02, where MSO-interpretations and unravelling of graphs are iterated, leading to an infinite hierarchy of graphs (or structures) with a decidable MSO-theory.

All the methods and results described so far can be separated into those concerned with FO logic (sometimes extended by a reachability relation [DT90, Col02]) and those dealing with MSO logic (sometimes with only restricted kind of set quantification as in [Mad03]). To our knowledge, there has been no systematic work on relating these two areas. In this paper, we bridge this gap by studying a new kind of interpretation, named finite sets interpretation, allowing to define classes of structures with decidable FO-theory from structures with decidable MSO-theory. To be more precise, we are considering weak MSO (WMSO) logic, i.e., MSO logic where quantification is restricted to finite sets. The idea for these interpretations is rather simple: the domain of the new structure does not consist of elements of the old structure but of finite sets of elements of the old structure. The relations are specified by WMSO-formulas with free set variables (the number of which corresponds to the arity of the relation). In this way, FO-formulas over the new structure can directly be translated into WMSO-formulas over the old structure. The use of WMSO ensures that the universe of the resulting structure is countable. It is not clear whether using standard MSO and then restricting to those resulting structures with a countable universe gives the same class of structures.

Using the equivalence of WMSO logic and finite automata (over finite words and trees) it is not difficult to see that the classes of automatic and tree automatic structures can be obtained by finite sets interpretations from $(\mathbb{N}, s u c c)$, i.e., the natural numbers with successor relation, and from the infinite binary tree, respectively (see [Col04]). The connection between automatic structures and finite sets interpretations applied to (N, succ) has already appeared before in the literature. In [ER66] the authors show that the infinite binary tree 
together with the equal level relation can be generated from $(\mathbb{N}, s u c c)$ by a finite sets interpretation. Today this extension of the infinite binary tree is known as a generator of automatic structures in the sense that every automatic structure can be obtained from it by a first-order interpretation. A similar result is given in [Rub04 but for another generator of the automatic structures.

This raises the question of what happens when we apply finite sets interpretations to other structures with decidable WMSO-theory, e.g., the structures from the hierarchy defined in Cau02. Though this hierarchy is strict, it is not a priori clear whether this is also true for the hierarchy obtained after applying finite sets interpretations. To answer questions of this kind one has to study the expressiveness of finite sets interpretations and to provide tools for showing that a structure cannot be obtained by such an interpretation applied to a given structure. In particular, such tools can then be used to answer questions on automatic structures because these can be obtained by finite sets interpretations (as mentioned above). More precisely, we concentrate ourselves in understanding what are the structures which are finite sets interpretatable in trees. All the examples mentionned so far fall in this category.

We contribute to this study via two results. The first one, Theorem 4.1, establishes that the quotient of a structure finite sets interpretable in a deterministic tree is itself finite sets interpretable in that tree. This result was known for automatic structures, and was open for tree-automatic structures. Theorem 4.1 is a generalisation of those two cases.

The second and main result, Theorem 4.3, allows to reduce questions on definability by finite sets interpretations to questions on WMSO-interpretability. A precise formulation of it (in its simplest form, see Corollary 4.5) reads as follows: If the class of structures definable by finite sets interpretations from a structure $\mathcal{S}$ is included in the class of structures definable by finite sets interpretations from a deterministic tree $t$, then $\mathcal{S}$ is WMSO-interpretable in $t$. Those questions of WMSO-interpretability in trees are well understood since they can be reformulated in terms of clique-width. The clique-width is a measure of the complexity of graphs which has been first introduced for finite graphs [Cou89], and then extended to infinite ones [Cou04]. In this latter case, the equivalence is expressible as follows: "a graph is WMSO-interpretable in a tree iff it is of bounded clique-width". Our result implies that if we can show that $\mathcal{S}$ is not WMSO-interpretable in $t$, then there are structures that can be obtained by a finite sets interpretation from $\mathcal{S}$ but not from $t$. A more technical formulation of the main result also explicitly gives such a structure.

We demonstrate the use of Theorems 4.1 and 4.3 by showing some non-definability results, the strictness of the hierarchy mentioned above, and a result on intrinsic definability of relations related to similar questions studied for automatic structures (cf. [Bár06]).

The remainder of the paper is structured as follows. In Section 2 we give the basic definitions and introduce finite sets interpretations, and in Section 3 we give the connection to automatic structures. Section 4 is devoted to the study of finite sets interpretations applied to trees. In particular, our two results, Theorems 4.1 and 4.3 are stated in this section. In Section 5 we present some applications of our results. Finally, Section 6 is devoted to the proof of the main result. 


\section{Definitions AND ElEMENTARY RESUlts}

In this section we provide the basic definitions used in the paper, i.e., relational structures, trees, logic, automata, and finally interpretations, the main subject of this work. We end this section by giving some elementary results on finite sets interpretations.

2.1. Structures and trees. We consider (relational) structures $\mathcal{S}=\left(\mathcal{U}, R_{1}, \ldots, R_{N}\right)$ where $\mathcal{U}$ is the universe of the structure and for each $i, R_{i} \subseteq \mathcal{U}^{r_{i}}$ is a relation of arity $r_{i}$ for a natural number $r_{i}$. The names of the $R_{i}$ together with their arities form the signature of the structure. Trees, as defined below, can be seen in a natural way as particular instances of such structures.

We will be dealing with infinite binary labeled trees. From now, we simply write 'trees'. Formally, a tree labeled by a finite alphabet $\Sigma$ is a partial mapping $t:\{0,1\}^{*} \rightarrow \Sigma$ with prefix closed domain $\operatorname{dom}(t)$, and such that if $u 1 \in \operatorname{dom}(t)$ then also $u 0 \in \operatorname{dom}(t)$. The elements of the domain are called nodes. A node $u$ such that $u 0$ is also a node is called an inner node, else it is called a leaf. By $\sqsubseteq$ we denote the prefix ordering on nodes, also called the ancestor order. For technical simplifications we will mostly consider purely binary trees, i.e., such that every node is either a leaf or has two sons.

Seen as a structure a tree labeled by $\Sigma$ has as universe the domain of the tree and contains the following relations: the unary relations $S_{0}$ and $S_{1}$ meaning 'being a left successor (resp. a right successor)' (for $i \in\{0,1\}, S_{i}(u, v)$ holds if $v=u i$ ) and for each $a \in \Sigma$ a unary relation $a$ interpreted as the set of elements sent to $a$ by $t$.

We will be considering two particular infinite trees, namely $\Delta_{1}$ and $\Delta_{2}$. The tree $\Delta_{1}$ is the unlabeled tree of domain $0^{*}$. We will identify in a natural way this tree with the structure $(\mathbb{N}$, succ $)$. The tree $\Delta_{2}$ is the unlabeled tree of domain $\{0,1\}^{*}$, also called the infinite binary tree.

2.2. Logic and automata. We use the standard definitions for first-order (FO) and weak monadic second-order (WMSO) logic. FO-formulas are built up from atomic formulas using first-order variables (interpreted by elements of the structure and usually denoted by letters $x, y, z)$ and the relation symbols from the signature under consideration. Complex formulas are constructed using boolean connectives and quantification over first-order variables.

For WMSO-formulas one can additionally use monadic second-order variables (interpreted by finite sets of elements of the structure and usually denoted by capital letters $X, Y, Z)$, quantification over such variables, and the membership relation $x \in X$. If the variables are interpreted by arbitrary sets instead of finite sets, then we speak of MSO.

In order to deal with WMSO-formulas on trees, we use automata. Those automata are more general than WMSO-formulas since they have the expressiveness of full MSO logic on trees. But for our purpose this doesn't harm because we only use the translation in one direction, namely from formulas to automata, and on deterministic trees one can define finiteness of a set in MSO (a set is finite if its prefix closure does not contain an infinite path), meaning that for each WMSO-formula there is an equivalent MSO-formula.

Technically, we use nondeterministic parity automata (or simply automata), which are tuples $\left(\Sigma, Q, q^{\text {in }}, \delta, \Omega\right)$ with a finite set $Q$ of states, initial state $q^{\text {in }}$, transition relation $\delta \subseteq Q \times$ $Q \times \Sigma \times Q \uplus \Sigma \times Q$, and priority mapping $\Omega: Q \rightarrow \mathbb{N}$. Recall that we only consider binary trees. Given a tree $t$ and an automaton, a run of this automaton on $t$ is a mapping $\rho: \operatorname{dom}(t) \rightarrow Q$ such that $(\rho(u 0), \rho(u 1), t(u), \rho(u)) \in \delta$ for each inner node $u$, and $(t(v), \rho(v)) \in \delta$ for every 
leaf $v$. A run is accepting if $\rho(\epsilon)=q^{\text {in }}$ and for all infinite branches (maximal totally ordered sequences of nodes) $v_{1}, v_{2}, \ldots, \liminf _{i} \Omega\left(\rho\left(v_{i}\right)\right)$ is even. We say that a tree $t$ is accepted by an automaton if there is an accepting run of this automaton on $t$. For basic properties of such automata (such as closure under the Boolean operations) and their relation to logic, we refer the reader to [Tho97.

We are interested here in automata running on a fixed underlying tree $t$ with additional markings representing (tuples of) subsets of its domain. To mark a certain subset $X$ of $\operatorname{dom}(t)$ we can put additional labels on the tree. Formally, the tree $t$ annotated by $X$ is the tree with the same domain as $t$ and labels from $\Sigma \times\{0,1\}$, where a node $u$ is labeled by the pair $(t(u), 0)$ if $u \notin X$ and $(t(u), 1)$ if $u \in X$. In the same way one can also annotate a tree with tuples of subsets of its domain using a separate $\{0,1\}$-component for each set.

If $t$ is fixed and $X_{1}, \ldots, X_{n}$ are subsets of $\operatorname{dom}(t)$, then we say that an automaton accepts the tuple $\left(X_{1}, \ldots, X_{n}\right)$ if it accepts $t$ with the additional labelings corresponding to the tuple $\left(X_{1}, \ldots, X_{n}\right)$ as explained above. If we consider all the tuples accepted by an automaton, we obtain a relation over the subsets of $\operatorname{dom}(t)$. We call this relation the relation recognized or accepted by the automaton.

A WMSO-formula with free set variables $X_{1}, \ldots, X_{n}$ also defines a relation over the subsets of $\operatorname{dom}(t)$. Throughout the paper we make use of the following result stating that for each WMSO-formula there is an equivalent automaton. The proof of this can easily be inferred from the equivalence of MSO and automata over trees and from the fact that over trees each WMSO-formula can be translated into an equivalent MSO-formula.

Theorem 2.1 (cf. [Tho97]). For each WMSO-formula there is an automaton such that for each tree $t$ the relation over subsets of dom $(t)$ defined by the formula is the same as the one accepted by the automaton.

2.3. Interpretations. Interpretations are a standard tool in logic allowing to define transformations of structures by means of logical formulas. This technique allows easy transfer of theories from one structure onto another.

Definition 2.2. An interpretation is a tuple $\left(\delta, \Phi_{R_{1}}, \ldots, \Phi_{R_{N}}\right)$ of formulas. The formula $\delta$ has only one free variable, and each formula $\Phi_{R_{i}}$ has $r_{i}$ free variables. By our convention, for weak monadic variables we use capital letters $X$ and $X_{1}, \ldots, X_{r_{i}}$, and small letters $x$ and $x_{1}, \ldots, x_{r_{i}}$ in the case of first-order variables.

An interpretation is FO if the formulas are first-order (and hence the free variables are also of first-order). An interpretation is WMSO if the formulas are weak monadic and the free variables are first-order. An interpretation is finite sets if the formulas are weak monadic and the free variables are weak monadic.

The application of an interpretation to a structure is defined in the standard way. The only difference is that for finite sets interpretations the elements of the obtained structure are finite subsets of the universe of the original structure instead of elements of the original structure. Formally, given a structure $\mathcal{S}$ and an interpretation $\mathcal{I}=\left(\delta, \Phi_{R_{1}}, \ldots, \Phi_{R_{N}}\right)$, the structure $\mathcal{I}(\mathcal{S})$ has for universe

- $\left\{u \in \mathcal{U}^{\mathcal{S}}: \mathcal{S} \models \delta(u)\right\}$ if $\mathcal{I}$ is a FO or WMSO interpretation,

- $\left\{U \subseteq \mathcal{U}^{\mathcal{S}}: U\right.$ finite, $\left.\mathcal{S} \models \delta(U)\right\}$ if $\mathcal{I}$ is a finite sets interpretation, 


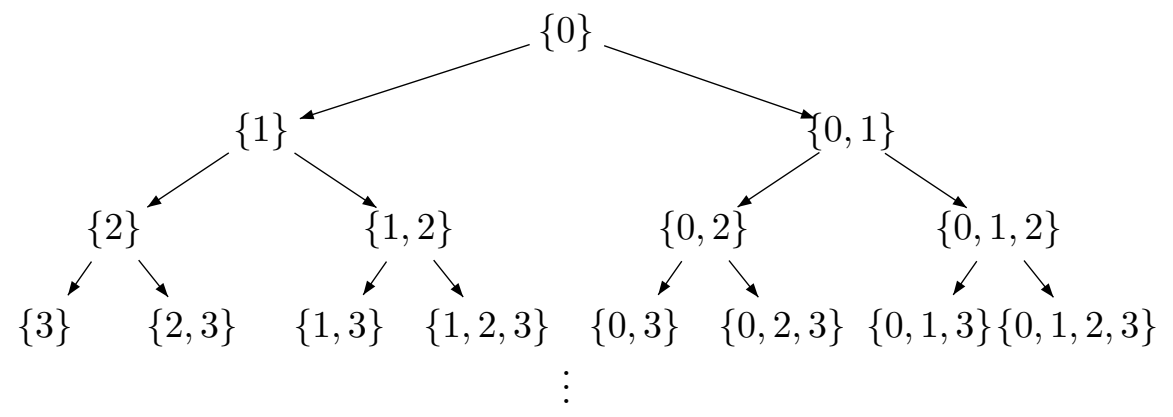

Figure 1: The nodes of the infinite binary tree $\Delta_{2}$ coded by sets of natural numbers

and the interpretation of each symbol $R_{i}$ is defined by

$$
R_{i}=\left\{\left(U_{1}, \ldots, U_{r_{i}}\right) \in\left(\mathcal{U}^{\mathcal{I}(\mathcal{S})}\right)^{r_{i}}: \mathcal{S} \models \Phi_{i}\left(U_{1}, \ldots, U_{r_{i}}\right)\right\} .
$$

One can note at this point that natural sets interpretations can as well be defined in a similar way, simply by removing the finiteness hypothesis on sets and using MSO instead of WMSO. At the end of this section we briefly comment on such possible variants for the definition.

The following example already appears in ER66. It shows how the complete binary tree extended with the equal level relation can be defined by a finite sets interpretation from the natural numbers with successor relation. This extension of the binary tree is well-known as a generator of the automatic structures, in the sense that each automatic structure can be obtained from it by an FO-interpretation (cf. BG00]).

Example 2.3. We show how to obtain the structure $\left(\{0,1\}^{*}, S_{0}, S_{1}\right.$,, el), i.e., the infinite binary tree extended with the prefix and equal level relations, by a finite sets interpretation from the infinite unary tree $\Delta_{1}$, i.e., from the natural numbers with successor $\Delta_{1}=(\mathbb{N}, s u c c)$. To realize this we have to code the nodes of the tree by finite sets of natural numbers and to describe the relations $S_{0}$ (for left successor), $S_{1}$ (for right successor), $\sqsubseteq$ (for prefix), and el (for equal level) by means of WMSO formulas.

The coding of the nodes is depicted in Figure1. A node $u \in\{0,1\}^{*}$ is represented by the set of positions corresponding to letter 1 in $u$ and additionally by its length. For example, the node 100 is coded by $\{0,3\}$ because its length is 3 and only position 0 is labeled 1 . We now define the finite sets interpretation $\mathcal{I}=\left(\delta, \Phi_{S_{0}}, \Phi_{S_{1}}, \Phi_{\sqsubseteq}, \Phi_{e l}\right)$ such that $\mathcal{I}\left(\Delta_{1}\right)$ yields the binary tree depicted in Figure 1 together with the relations $\sqsubseteq$ and $e l$. In the formulas we use abbreviations like $<$ and max that can easily be defined by WMSO-formulas in $\Delta_{1}$.

- $\delta(X):=\exists x(x \in X)$ (all finite sets except $\emptyset$ are used in the coding).

- $\Phi_{\sqsubseteq}\left(X_{1}, X_{2}\right):=\max \left(X_{1}\right)<\max \left(X_{2}\right) \wedge \forall x\left(x<\max \left(X_{1}\right) \rightarrow\left(x \in X_{1} \leftrightarrow x \in X_{2}\right)\right)$.

- $\Phi_{S_{0}}\left(X_{1}, X_{2}\right):=\Phi_{\sqsubseteq}\left(X_{1}, X_{2}\right) \wedge \max \left(X_{2}\right)=\max \left(X_{1}\right)+1 \wedge \max \left(X_{1}\right) \notin X_{2}$.

- $\Phi_{S_{1}}\left(X_{1}, X_{2}\right):=\Phi_{\sqsubseteq}\left(X_{1}, X_{2}\right) \wedge \max \left(X_{2}\right)=\max \left(X_{1}\right)+1 \wedge \max \left(X_{1}\right) \in X_{2}$.

- $\Phi_{e l}\left(X_{1}, X_{2}\right):=\max \left(X_{1}\right)=\max \left(X_{2}\right)$

Let us proceed with some elementary considerations. Obviously, finite sets interpretations are not closed under composition. But, as stated in the following proposition, applying 
an FO-interpretation after, or a WMSO-interpretation before a finite sets interpretation, does not give more expressive power.

Proposition 2.4. Let $\mathcal{I}_{1}$ be a FO-interpretation, $\mathcal{I}_{2}$ be a WMSO-interpretation, and $\mathcal{I}$ be a finite sets interpretation. Then $\mathcal{I}_{1} \circ \mathcal{I} \circ \mathcal{I}_{2}$ is effectively a finite sets interpretation.

Proof. As for standard interpretations.

A straightforward as well as essential consequence of this is expressed in the following corollary.

Corollary 2.5. The image of a structure of decidable WMSO-theory by a finite sets interpretation has a decidable FO-theory.

To finish our elementary considerations on finite sets interpretations, we present Proposition 2.7 which is a form of converse to Proposition 2.4. It states that every finite sets interpretation can be described as the composition of a specific one, called the weak powerset interpretation, and a first-order interpretation.

Definition 2.6. Let $\mathcal{P}^{W}$ be the finite sets interpretation that sends every structure $\mathcal{S}$ of signature $\Sigma$ onto a structure of signature $\Sigma \cup\{\preceq\}$ where $\preceq$ is a new binary symbol, such that

- the universe is the set of finite subsets of the universe of $\mathcal{S}$,

- each symbol $R$ in $\Sigma$ has the same interpretation as in $\mathcal{S}$ but over singletons instead of elements,

- the interpretation of $\preceq$ corresponds to the subset ordering.

The interpretation $\mathcal{P}^{W}$ is called the weak powerset interpretation.

This interpretation allows to reconstruct all other finite sets interpretations as stated in the following proposition which is obtained by a simple syntactic translation of formulas.

Proposition 2.7. For each finite sets interpretation $\mathcal{I}$ there exists a FO-interpretation $\mathcal{I}_{1}$ such that $\mathcal{I}_{1} \circ \mathcal{P}^{W}=\mathcal{I}$.

Possible variants. The definition of finite sets interpretations that we provide uses WMSO and one might wonder why we restrict ourselves to this logic. At least two modifications of the definition are very natural:

- replace WMSO logic with MSO logic in the formulas (but still only consider finite sets for the free variables),

- use full MSO and produce a structure over the powerset of the original structure instead of its finite subsets.

If we use the first extension, then Proposition 2.7 fails. All other results in this paper would remain unchanged.

The second extension leads to what can be naturally called sets interpretation. The formulas are MSO, as well as the free variables. When applying such a sets interpretation to a structure, one produces a structure of universe included in the powerset of the universe of the original structure.

All the results presented above remain valid for this variant. However, all the results developed below in this work make explicit use of the finiteness of the sets representing elements of the new structure. In particular, one can conjecture that Theorem 4.1 would 
fail for sets interpretations. While the conjecture would be that Theorem 4.3 still holds for sets interpretations.

This new kind of interpretation allows to define structures of uncountable universe from structures of countable universe. This makes a major difference with respect to finite sets interpretations. In general, the relation between set interpretations and finite set interpretations is not yet understood. In particular, we do not know whether the countable structures obtainable by sets interpretations from trees coincide with the structures obtainable by finite sets interpretations from trees.

\section{Automatic-Like structures}

The line of research that has inspired finite sets interpretations is the one of automatic structures. Automatic structures in their common acceptation are structures with a universe consisting of a regular language of words, and the relations defined by half-synchronized transducers. The key reason for introducing such structures is that - thanks to the good closure properties of finite automata - they naturally possess decidable first-order theories.

Classical variants of those structures consider universes consisting of infinite words $(\omega-$ automatic structures), or consisting of trees, finite or infinite (namely the tree-automatic and $\omega$-tree-automatic structures). Some definitions also allow to quotient the structure by a congruence (that is defined in the same way as the other relations). This extension does not increase the expressiveness of automatic nor tree-automatic structures (cf. Corollary 4.2 below). The question whether quotienting increases the expressive power of $\omega$-automatic and $\omega$-tree-automatic structures is open.

Historically, automatic as well as $\omega$-automatic structures have been introduced by Hodgson [Hod83. Khoussainov and Nerode introduce the notion of automatically presentable theories [KN95], starting the study of definability in automatic structures. The extension to tree-automatic structures can be traced back, in a different framework, to the work of Dauchet and Tison [DT90]. Blumensath and Grädel Blu99, BG00 then formalize the notion of tree-automatic structure and add to it the family of $\omega$-tree-automatic structures. Independently, the study of 3-manyfolds lead to the particular case of automatic groups $\left[\mathrm{ECH}^{+} 92\right]$.

3.1. Word-automatic structures. In the case of words a relation $R \subseteq\left(\Sigma^{*}\right)^{r}$ is automatic if there is a finite automaton accepting exactly the tuples $\left(w_{1}, \ldots, w_{r}\right) \in R$, where the automaton reads all the words in parallel with the shorter words padded with a dummy symbol $\diamond$. Formally, for $w_{1}, \ldots, w_{r} \in \Sigma^{*}$ we define

$$
w_{1} \otimes \cdots \otimes w_{r}=\left[\begin{array}{c}
a_{11}^{\prime} \\
\vdots \\
a_{r 1}^{\prime}
\end{array}\right] \cdots\left[\begin{array}{c}
a_{1 n}^{\prime} \\
\vdots \\
a_{r n}^{\prime}
\end{array}\right] \in\left(\Sigma_{\diamond}^{r}\right)^{*}
$$

where $\Sigma_{\diamond}=\Sigma \cup\{\diamond\}, n$ is the maximal length of one of the words $w_{i}$, and $a_{i j}$ is the $j$ th letter of $w_{i}$ if $j \leq\left|w_{i}\right|$ and $\diamond$ otherwise. A language $L \subseteq\left((\Sigma \cup\{\diamond\})^{r}\right)^{*}$ defines a relation $R_{L} \subseteq\left(\Sigma^{*}\right)^{r}$ in the obvious way: $\left(w_{1}, \ldots, w_{r}\right) \in R_{L}$ iff $w_{1} \otimes \cdots \otimes w_{r} \in L$. A tuple $\left(L, L_{1}, \ldots, L_{n}\right)$ of languages $L \subseteq \Sigma^{*}$ and $L_{i} \subseteq\left(\Sigma_{\diamond}^{r_{i}}\right)^{*}$ defines a structure of universe $L$ with the relations $R_{L_{i}}$ of arity $r_{i}$. A structure $\mathcal{S}=\left(\mathcal{U}, R_{1}, \ldots, R_{n}\right)$ is automatic if it is isomorphic to a structure of the above kind for regular languages $L, L_{1}, \ldots, L_{n}$. 
The class of $\omega$-automatic structures is defined in the same way with infinite words instead of finite ones. In this case the definition is even simpler as there is no need for padding shorter words.

3.2. Tree-automatic structures. To define tree-automatic structures we need a way to code tuples of finite trees, i.e., we need an operation $\otimes$ for finite trees. For a tree $t$ : $\operatorname{dom}(t) \rightarrow \Sigma$ let $t^{\diamond}:\{0,1\}^{*} \rightarrow \Sigma_{\diamond}$ be defined by $t^{\diamond}(u)=t(u)$ if $u \in \operatorname{dom}(t)$, and $t^{\diamond}(u)=\diamond$ otherwise. For finite $\Sigma$-labeled trees $t_{1}, \ldots, t_{r}$ we define the $\Sigma_{\diamond}^{r}$-labeled tree $t=t_{1} \otimes \cdots \otimes t_{r}$ by $\operatorname{dom}(t)=\operatorname{dom}\left(t_{1}\right) \cup \cdots \cup \operatorname{dom}\left(t_{r}\right)$ and $t(u)=\left(t_{1}^{\diamond}(u), \ldots, t_{r}^{\diamond}(u)\right)$. When viewing words as unary trees, this definition corresponds to the operation $\otimes$ as defined for words. As in the case of words a set $T$ of finite $\Sigma_{\diamond}^{r}$-labeled trees defines the relation $R_{T}$ by $\left(t_{1}, \ldots, t_{r}\right) \in R_{T}$ iff $t_{1} \otimes \cdots \otimes t_{r} \in T$. A structure is called tree-automatic if it is isomorphic to a structure given by a tuple $\left(T, T_{1}, \ldots, T_{n}\right)$ of regular tree languages in the same way as for words.

Again, the definitions for $\omega$-tree-automatic structures are the same with $\omega$-trees, i.e., trees of domain $\{0,1\}^{*}$ instead of finite trees.

One should note here that we only consider so called injective presentations of automatic structures. A more general definition as, e.g., in [Blu99] additionally uses a regular language $L_{\sim} \subseteq\left(\Sigma_{\diamond}^{2}\right)^{*}$ defining an equivalence relation identifying words representing the same element of the structure (and similarly for the other variants of automatic structures). It is known that injective presentations are sufficient for automatic structures [KN95] meaning that all structures that are automatic in the more general sense are also automatic according to our definition. The corresponding result for tree-automatic structures is established below, see Corollary 4.2 .

3.3. Automaticity via interpretations. Recall that $\Delta_{1}$ is the (unlabeled) infinite unary tree, i.e., the natural numbers with successor, and that $\Delta_{2}$ is the (unlabeled) infinite binary tree. The following fact is a straightforward consequence of the definition of automatic structures and of the equivalences between WMSO-logic and automata. The first claim also appears in [Rub04, Thm. C.2.11, page 50].

Proposition 3.1. The following holds up to isomorphism

- A structure is automatic iff it is finite sets interpretable in $\Delta_{1}$.

- A structure is tree-automatic iff it is finite sets interpretable in $\Delta_{2}$.

Proof (sketch). As already mentioned, the first claim is shown in [Rub04].

We describe here how to proceed for tree-automatic structures, starting by an explanation how to obtain a tree-automatic presentation from a finite sets interpretation $\mathcal{I}$ in $\Delta_{2}$. A finite subset $X$ of $\Delta_{2}$ is coded as a finite tree as follows: the tree has the smallest domain that contains all elements from $X$ and is labeled 0 at positions that are not in $X$ and 1 at positions that are in $X$. For each formula of $\mathcal{I}$ there is an equivalent parity automaton over $\Delta_{2}$. This automaton can easily be turned into an automaton over finite trees accepting the corresponding relation over the codings as just described.

For the other direction we start from a tree-automatic presentation of a structure. A first thing to note is that a singleton alphabet of tree labels is sufficient because each symbol from a larger alphabet can be encoded in a finite pattern; in this construction, we use the leaf/non-leaf nature of each node for coding information. Since now the alphabet is a singleton, one naturally encodes a tree $t$ by the finite set $d o m(t)$. Now pick an automaton 
from the tree-automatic presentation accepting a relation and pick a tuple of such sets coding finite trees. Using standard techniques for translating automata to logic (cf. [Tho97]), one describes in WMSO that the corresponding tuple of finite trees is accepted by the automaton.

Note that the $\omega$-automatic and $\omega$-tree-automatic structures satisfy the same equivalences where finite sets interpretations are replaced by sets interpretations.

\section{Finite Sets interpretations on trees}

The power of finite sets interpretations makes it difficult to obtain results for the general case where such interpretations are applied to arbitrary structures. For this reason, in this article we only consider the special case of finite sets interpretations applied to deterministic trees.

This restriction can be justified in two ways. The first justification is that on trees there are specific tools suitable for treating WMSO questions: their automata equivalents. The second justification is that if Seese's conjecture [See91] holds - stating that all structures of decidable weak monadic second-order theory are WMSO-interpretations of trees - then the only structures that we can prove to have decidable first-order theory using Corollary 2.5 are finite sets interpretations of trees (for recent work on Seese's conjecture see [CO06]).

We give here two results concerning finite sets interpretations applied to deterministic trees. The first one - in Subsection 4.1 - shows that finite sets interpretations on deterministic trees followed by a quotient are simply finite sets interpretations. The second result - subject of Subsection 4.2 - concerns finite sets interpretations applied to trees leading to powerset lattices. The technical core of the proof is given in Section 6 ,

4.1. Quotienting finite sets interpretations on trees. We show here that if a structure is finite sets interpretable in a deterministic tree containing a symbol interpreted as a congruence on the structure, then it is possible to directly obtain the quotiented structure by a finite sets interpretation.

A congruence on a structure $\mathcal{S}$ is an equivalence relation $\sim$ such that for every symbol $R$ of arity $n$ and all elements $x_{1}, \ldots, x_{n}, y_{1}, \ldots, y_{n}$ of $\mathcal{S}:$ if $x_{1} \sim y_{1}, \ldots, x_{n} \sim y_{n}$, then $R^{\mathcal{S}}\left(x_{1}, \ldots, x_{n}\right)$ iff $R^{\mathcal{S}}\left(y_{1}, \ldots, y_{n}\right)$. We say that a symbol is a congruence if its interpretation in the structure is a congruence. For a congruence $\sim$ over a structure $\mathcal{S}$, we denote by $\mathcal{S} / \sim$ the quotient structure, i.e., the structure which has as elements the equivalence classes of $\sim$, and the relations of which are the images of the relations on $\mathcal{S}$ under the canonical surjection induced by $\sim$.

Note that the operation of quotienting preserves the decidability of the first-order theory. For this reason we may wonder if constructing a structure by a finite sets interpretation followed by a quotient is more powerful than solely using a finite sets interpretation. Theorem 4.1 below shows that it is not the case when the original structure is a deterministic tree.

Theorem 4.1. Given a finite sets interpretation $\mathcal{I}$, there exists a finite sets interpretation $\mathcal{I}^{\prime}$ such that for every deterministic tree $t$, if the symbol $\sim$ is a congruence in $\mathcal{I}(t)$, then $\mathcal{I}^{\prime}(t)$ is isomorphic to $\mathcal{I}(t) / \sim$. 
Proof. Let $\mathcal{A}$ be a nondeterministic parity automaton corresponding to the formula $\Phi_{\sim}$ describing $\sim$ in $\mathcal{I}$. This automaton works on $t$ additionally annotated by a pair $(X, Y)$ of sets of nodes. We say that the automaton reads $(X, Y)$.

For a prefix closed subset $S$ of $\operatorname{dom}(t)$, we call the set of minimal nodes not in $S$ its border. Let $X$ be an element of the structure $\mathcal{I}(t)$, i.e., a finite set of nodes of $t$. We construct its shadow $S(X)$ by taking the prefix closure of $X$ and then adding all trees of height at most $2^{|Q|}-1$ that are rooted at the border of this prefix closure. So $S(X)$ is the least set of nodes containing $X$ that is closed by prefix and such that all element of its border is the root of a subtree of height at least $2^{|Q|}$. Obviously, $S(X)$ is finite. Now, let us consider an equivalence class $c$ for $\sim$. Define the shadow $S(c)$ of the class $c$ to be the intersection of the shadows of all the elements in the class. This is also a finite set of nodes of $t$. We define the border of the class, denoted by $B(c)$, to be the border of its shadow. Note that the subtrees rooted at nodes from $B(c)$ have height at least $2^{|Q|}$; indeed, this property is preserved when intersecting shadows.

Given an element $X$, its description is the triple $(B, Y, f)$, where $B=B(c)$ for the class $c$ of $X, Y$ is $X \cap S(c)$, i.e., $X$ restricted to the shadow of its class, and $f$ maps each node $x \in B$ to the set of states $q$ such that there is an accepting run of $\mathcal{A}$ on the subtree rooted in $x$ starting with state $q$ and reading $(\emptyset, X)$.

We claim that if two elements $X$ and $X^{\prime}$ share the same description - say $(B, Y, f)-$ then those elements are equivalent for $\sim$. Using the transitivity of $\sim$ and the finiteness of $B$ it is sufficient to consider the case of elements coinciding everywhere but below one $x$ in $B$. Note that $X$ and $X^{\prime}$ coincide above $B$ because they have the same description. Since $x$ is in the border of the class of $X$, there is an element $Z$ equivalent to $X$ such that $Z$ does not contain any node below $x$. Since $Z$ is equivalent to $X$, there is an accepting run $\rho$ of $\mathcal{A}$ on $t$ labeled by $(Z, X)$. We aim at constructing a run of $\mathcal{A}$ witnessing the equivalence of $Z$ and $X^{\prime}$, i.e., a run accepting $t$ labeled by $\left(Z, X^{\prime}\right)$. This new run is constructed in the following way. On every element not below $x$, it coincides with $\rho$. This is a valid part of run since $X$ and $X^{\prime}$ do coincide on this area. On the subtree rooted in $x, Z$ coincides with $\emptyset$. Hence, as $(B, Y, f)$ is a description of $X, \rho(x)$ belongs to $f(x)$. But as the same description holds also for $X^{\prime}$, there is a piece of run below $x$ starting with $\rho(x)$ and accepting $\left(\emptyset, X^{\prime}\right)$. We complete our new run by this piece of run. This new run witnesses as expected that $Z \sim X^{\prime}$. It follows by symmetry and transitivity of $\sim$ that $X \sim X^{\prime}$. This concludes the proof of the claim.

Let us remark now that a description $(B, Y, f)$ can be encoded uniquely by a set of nodes: this set is $B \cup Y \cup \operatorname{Coding}(f)$ where $\operatorname{Coding}(f)$ contains exactly one element for each element $x$ of the border, and this element is located in a place uniquely describing the value of $f(x)$ (e.g. the leftmost node at distance $g(f(x))$ below $x$, where $g$ is a numbering of $2^{Q}$ starting from 1). This is possible since the subtree rooted in $x$ is has height at least $2^{|Q|}$, and consequently, there is "room" below $x$ for coding the information $f(x)$.

Note that associating to an element $X$ the coding of its description is doable by means of a WMSO-formula. Note also that given a class $c$ there is only a finite number of descriptions for the elements it contains. Hence we can choose the smallest description - smallest for a suitable total order - as unique representative for the class. A suitable total order can, e.g., use the lexicographically smallest node that is in the symmetric difference of the two sets coding the two descriptions. From here, it is not difficult to reconstruct $\mathcal{I}(t) / \sim$.

In combination with Proposition 3.1 we obtain the following. 
Corollary 4.2. Tree-automatic structures are effectively closed under quotient.

In the terminology of [Blu99], this result is rephrased as "every tree-automatic structure admits an injective presentation." Let us remark that this result is announced in [Blu99], but unfortunately the proof proposed there contains an unrecoverable error.

4.2. Finite sets interpretations and powerset lattices. In this section we present our main result. For this, let $\mathcal{S}$ be a structure of signature $\preceq$. We say that $\mathcal{S}$ is a finite powerset lattice if it is isomorphic to $\left(\mathcal{P}^{F}(E), \subseteq\right)$ for some set $E$, where $\mathcal{P}^{F}(E)$ represents the finite subsets of $E$. Such a finite powerset lattice can be seen as a particular case of weak powerset generator applied to a vocabulary-free structure. We call the elements corresponding to singletons in this isomorphic structure atoms, i.e., those elements which have exactly one element strictly smaller with respect to $\preceq$.

Theorem 4.3. For every finite sets interpretation $\mathcal{I}=\left(\delta(X), \phi_{\preceq}(X, Y)\right)$, there exists a $W M S O$-formula Code $(X, x)$ such that, whenever for some tree $t, \overline{\mathcal{I}}(t)$ is a finite powerset lattice, then Code $(X, x)$ evaluates on $t$ to an injection mapping the atoms of $\mathcal{I}(t)$ to nodes of $t$.

Section 6 is dedicated to the rather long and involved proof of this result.

We rarely use the theorem in this form. We rather use weakened versions of it, namely Corollary 4.4 and Corollary 4.5 .

Corollary 4.4. For every finite sets interpretation $\mathcal{I}$, there exists a WMSO-interpretation $\mathcal{I}_{2}$ such that whenever for some structure $\mathcal{S}$ and some tree $t, \mathcal{I}(t)$ is isomorphic to $\mathcal{P}^{W}(\mathcal{S})$ then $\mathcal{I}_{2}(t)$ is isomorphic to $\mathcal{S}$.

Proof. If we remove all relations other than $\preceq$, the weak powerset generator is nothing but a finite powerset lattice. Hence we can obtain a formula $\operatorname{Code}(X, x)$ by application of Theorem 4.3. It is then easy to transfer all relations defined on singletons to their image by Code.

Formally, we define the WMSO-interpretation $\mathcal{I}_{2}=\left(\delta, \Phi_{R_{1}}, \ldots, \Phi_{R_{l}}\right)$ as follows:

- $\delta(x)=\exists X$.Code $(X, x)$,

- for each symbol $R$ of arity $r$ from the signature, $\Phi_{R}\left(x_{1}, \ldots, x_{r}\right)$ is defined as

$$
\exists X_{1}, \ldots, X_{r} . \Psi_{R}\left(X_{1}, \ldots, X_{r}\right) \wedge \bigwedge_{i=1}^{r} \operatorname{Code}\left(X_{i}, x_{i}\right)
$$

where each $\Psi_{R}$ is the WMSO-formula in $\mathcal{I}$ defining the interpretation of the symbol $R$.

As Code maps each atom of $\mathcal{I}(t)$ to a unique node, this interpretation indeed maps $t$ to a structure isomorphic to $\mathcal{S}$.

A weaker, yet more readable formulation of the above corollary is provided in the following one.

Corollary 4.5. If for a structure $\mathcal{S}$ and a tree $t$ the class of structures that can be obtained by finite sets interpretations from $\mathcal{S}$ is contained in the class of structures that can be obtained by finite sets interpretations from $t$, then $\mathcal{S}$ is WMSO-interpretable in $t$.

Proof. Assume that the hypothesis of the corollary holds. In particular, the structure $\mathcal{P}^{W}(\mathcal{S})$ is isomorphic to $\mathcal{I}(t)$ for some finite sets interpretation $\mathcal{I}$. By applying Corollary 4.4, the structure $\mathcal{S}$ is WMSO-interpretable in $t$. 


\section{Applications}

We present here several applications of the results above, ordered by level of complexity. The two first ones, showing that the free monoid is not obtainable by a finite sets interpretation of a tree (Section 5.1) and that a natural hierarchy of structures is strict (Section 5.2), are paradigmatic applications of Theorem 4.3. Section 5.3 establishes that the random graph is not finite sets interpretable in a tree, extending the known result for automatic structures KNRS04. Finally, in Section 5.4, we study intrinsically definable relations in generators of the automatic structures.

Some of those results are known in the weaker context of automatic structures. We would like to underline here the fundamental methodological difference of our approach: in none of the applications below we perform a combinatorial analysis of finite sets interpretations. Instead, we systematically reduce the problem to a much easier one of WMSOdefinability in trees.

5.1. The free monoid. We consider the free monoid as a structure $\left(\{a, b\}^{*}, \cdot, a, b\right)$ - the set of words over $\{a, b\}$ together with the ternary relation corresponding to the concatenation and the two words $a$ and $b$ identified by unary predicates - and want to answer the question whether this structure is isomorphic to a finite sets interpretation of a tree.

One should note that the FO theory of the free monoid is undecidable and hence we can directly conclude that it cannot be obtained by a finite sets interpretation from a tree with a decidable WMSO theory. However, this reasoning does not include trees with an undecidable WMSO theory.

The negative answer we give here to the above question is the simplest and in some sense the purest application of the results presented above and should be considered for this reason as a key example.

The following result was obtained in a discussion with Olivier Ly.

Proposition 5.1. The free monoid over a two letter alphabet is not isomorphic to any finite sets interpretation of a tree.

Proof. We first show how to obtain $\mathcal{P}^{W}(\mathbb{N},+)$ from the free monoid by an FO-interpretation followed by a quotient. Then, assuming that our claim is false, we invoke the two results from the previous section and obtain a contradiction.

Let $f$ be the function which to each word of the form $b a^{n_{1}} b a^{n_{2}} b \ldots b a^{n_{k}} b$ over $\{a, b\}$ associates the set of naturals $\left\{n_{1}, n_{2}, \ldots, n_{k}\right\}$. The domain of $f$ is the set of elements satisfying $\operatorname{dom}(x)=\exists y \cdot x=b y b$. The relation of inclusion is also first-order definable: $f(u) \subseteq f(v)$ iff $\operatorname{sub}(u, v)$ holds with $\operatorname{sub}(u, v)=$

$$
\begin{gathered}
\forall x \in a^{*} \cdot \exists y, z . u=y b x b z \rightarrow \exists y^{\prime}, z^{\prime} \cdot v=y^{\prime} b x b z^{\prime}, \\
\text { where } x \in a^{*} \text { stands for } \forall y, z \cdot x \neq y b z .
\end{gathered}
$$

Let $x \sim y$ be the formula $s u b(x, y) \wedge s u b(y, x)$. Naturally, for every $u, v, u \sim v$ iff $f(u)=f(v)$. Finally the addition over singletons is definable. More precisely, $f(u)=\{i\}, f(v)=\{j\}$ and $f(w)=\{i+j\}$ iff $\operatorname{add}(u, v, w)$ holds with $\operatorname{add}(u, v, w)=$

$$
\exists x \in a^{*}, y \in a^{*} . u \sim b x b \wedge v \sim b y b \wedge w \sim b x y b .
$$

Using those formulas, one can first-order interpret in the free monoid a structure which, when quotiented by $\sim$, is isomorphic to $\mathcal{P}^{W}(\mathbb{N},+)$. 
Assume now that the free monoid can be finite sets interpreted in some tree $t$. Since structures obtainable by finite sets interpretations from $t$ are closed under first-order interpretations (Proposition 2.4) and quotient (Theorem 4.1), this implies that $\mathcal{P}^{W}(\mathbb{N},+$ ) is finite sets interpretable in $t$. By Corollary 4.4 we deduce that $(\mathbb{N},+)$ is WMSO-interpretable in $t$. This yields a contradiction since $(\mathbb{N},+)$ is not WMSO-interpretable in a tree. Let us provide a direct argument for proving this last argument.

Assume that $(\mathbb{N},+)$ is WMSO-interpretable in $t$ and let $U$ denote the set of nodes of $t$ that represent $\mathbb{N}$ in a corresponding interpretation. We first note that for each node of $t$ at most one of its subtrees contains infinitely many elements from $U$. Otherwise, if the two subtrees of a node $u$ contain both infinitely many elements from $U$, the successor relation on $\mathbb{N}$ (which is addition with one argument fixed to 1 ) would infinitely often jump between these two subtrees. If $\mathcal{A}$ is an automaton with $n$ transitions accepting the successor relation, and if $x_{0}, \ldots, x_{n} \in U$ are in the left subtree of $u, y_{0}, \ldots, y_{n} \in U$ are in the right subtree of $u$, and all $x_{i}, y_{i}$ are in the successor relation, then $\mathcal{A}$ also accepts a pair $x_{i}, y_{j}$ with $i \neq j$ by a simple counting argument on the transitions used at $u$ in accepting runs of $\mathcal{A}$.

By starting at the root and always proceeding to the unique subtree containing infinitely many elements from $U$ we obtain an infinite branch $B$ of $t$.

Now let $\mathcal{A}_{+}$be an automaton with $n$ transitions accepting the relation + on $t$ and let $x_{0}, \ldots, x_{n} \in U$. For each $x_{i}$ there are infinitely many $y_{i}, z_{i}$ such that the triple $\left(x_{i}, y_{i}, z_{i}\right)$ is accepted by $\mathcal{A}_{+}$. Choose a node $v$ on $B$ such that none of the $x_{i}$ is below $v$ and for each $i$ choose $y_{i}, z_{i}$ as above that are below $v$. Counting the possible transitions that are used at $v$ in accepting runs of $\mathcal{A}_{+}$on the tuples $\left(x_{i}, y_{i}, z_{i}\right)$ we obtain that $\mathcal{A}_{+}$also accepts $\left(x_{i}, y_{j}, z_{j}\right)$ for some $i \neq j$. This gives a contradiction.

5.2. A hierarchy of structures of decidable first-order theory. Caucal [Cau02] introduces a hierarchy of graphs/structures of decidable MSO-theory. The definition of this hierarchy that we use here differs from the original one and follows [CW03] and [Tho03.

Level 0 consists of finite structures, and level $n+1$ is defined as the MSO-interpretations of the unraveling of graphs of level $n$. As both MSO-interpretation and unraveling are transformations preserving the decidability of the MSO-theory, each structure of this hierarchy has a decidable MSO-theory. In [CW03, this hierarchy is shown to be strict.

If in these definitions the MSO-interpretations are replaced by WMSO-interpretations, we obtain the same hierarchy. This can be deduced from a result in CW03. stating that each graph of level $n$ can be obtained from the unravelling of a deterministic graph of level $n-1$ by applying a so called inverse rational mapping. Such an inverse rational mapping can easily be described by a WMSO-interpretation.

Furthermore, the unravelling of a deterministic graph yields a deterministic tree and on deterministic trees every WMSO-formula is equivalent to an MSO-formula. Hence, from the decidability of the MSO theory we also obtain the decidability of the WMSO theory for deterministic trees. In combination with the above mentioned result all graphs in the hierarchy have a decidable WMSO theory.

From this hierarchy, it is easy to construct a corresponding tree-automatic hierarchy. The tree-automatic structures of level $n$ are the image of the structures of level $n$ of the Caucal hierarchy by finite sets interpretations. Let us denote the $n$th level of this treeautomatic hierarchy by $\mathrm{TAUT}_{n}$. Since the trees on the first level of the Caucal hierarchy 
are regular, we can deduce from Proposition 3.1 that TAUT 1 coincide with the class of tree-automatic structures.

Furthermore, from Corollary 2.5 and the above considerations we can conclude the following decidability result.

Remark 5.2. For each $n$, every structure in $\mathrm{TAUT}_{n}$ has a decidable FO theory.

A simple application of our result is the strictness of this tree-automatic hierarchy.

Theorem 5.3. For each $n \geq 0$ the class TAUT $T_{n}$ of structures on the $n$th level of the automatic hierarchy is strictly contained in TAUT $T_{n+1}$.

Proof. We know that each level $n$ of the Caucal hierarchy contains a tree generator $G_{n}$, i.e., each structure of level $n$ is WMSO-interpretable in $G_{n}$ CW03. Let us suppose that the automatic hierarchy collapses at some level $n$, i.e., $\mathrm{TAUT}_{n}=\mathrm{TAUT}_{n+1}$. This would imply that the structure $\mathcal{P}^{W}\left(G_{n+1}\right)$ can be obtained by a finite sets interpretation from $G_{n}$. Then, by Corollary 4.5, we obtain $G_{n+1}$ as a WMSO-interpretation of $G_{n}$ and hence $G_{n+1}$ is in the $n$th level of the hierarchy. This contradicts the strictness of the Caucal hierarchy.

5.3. Random graph. The random graph is a non-oriented unlabeled countable graph with the following fundamental property: for any two disjoint finite set of vertices $E$ and $F$, there exists a vertex $v$ that is connected to all the elements of $E$ and to none of the elements of $F$. For the existence and basic properties of such a graph see, e.g., Hod93. We do not give in this work a more precise definition of the random graph. Anyhow, a direct consequence of the fundamental property stated above is that the random graph satisfies the quantifier elimination property (and this is effective). The decidability of its first-order theory follows.

Since the random graph has a decidable first-order theory and since finite sets interpretations define a large number of structures also having this property, it is interesting to consider the question whether the random graph can be obtained by a finite sets interpretation from a tree. A partial answer to this question has been studied: one knows that the random graph is not isomorphic to any word-automatic structure KNRS04.

In this section, we show that there is no tree from which the random graph can be generated by a finite sets interpretation. This proof was obtained in a discussion with Vince Bárány.

Theorem 5.4. The random graph is not finite sets interpretable in a tree.

Proof. Heading for contradiction, let us assume that there exists a finite sets interpretation $\mathcal{I}_{R}=(\delta(X), \Psi(X, Y))$ and a binary tree $t_{R}$ such that $\mathcal{I}_{R}\left(t_{R}\right)$ is (isomorphic to) the random graph.

The basic idea is to prove that, under this assumption, "the random graph is WMSOinterpretable in a tree". However, this statement is uncomfortable to handle since the properties of the random graph do only refer to its finite induced subgraphs. Instead, we show a similar interpretability result for every finite induced subgraph of the random graph; i.e. for every finite graph. Formaly we establish the following claim.

Claim: There exists a finite sets interpretation $\mathcal{I}^{\prime}$ such that for any finite non-oriented graph $G$ there exists a tree $t_{G}$ such that $\mathcal{I}^{\prime}\left(t_{G}\right)$ is isomorphic to $\mathcal{P}^{W}(G)$.

Before we prove this claim, let us demonstrate how to use it to show Theorem 5.4. Let us apply Corollary 4.4 on the interpretation $\mathcal{I}^{\prime}$. We obtain a WMSO-interpretation $\mathcal{I}^{\prime \prime}$ with 
the property that $\mathcal{I}^{\prime \prime}\left(t_{G}\right) \cong G$ for any non-oriented unlabeled graph $G$ and a suitably chosen tree $t_{G}$. As trees have bounded clique-width and WMSO-interpretations applied to a class of graphs of bounded clique-width yield also a class of graphs of bounded clique-width (see e.g. Cou97] , we obtain a contradiction to the fact that there exists non-oriented graphs of arbitrary high clique-width (the $(n \times n)$-grids yield such a family of graphs, see [MR99]).

Proof of the claim: Let us show first how we can encode any finite set of elements of $\mathcal{I}_{R}\left(t_{R}\right)$ by a pair of finite subsets of $d o m\left(t_{R}\right)$ in such a way that the membership relation is "definable".

Let $E$ be a finite set of vertices of $\mathcal{I}_{R}\left(t_{R}\right)$. Each vertex of $\mathcal{I}_{R}\left(t_{R}\right)$ is a finite set of nodes of $t_{R}$ and therefore it makes sense to define $D_{E}$ as the union of all the elements in $E$. Furthermore, let us chose $I_{E}$ to be an element of $\mathcal{I}_{R}\left(t_{R}\right)$ which is connected to all elements of $E$ and to none of the elements of $\mathcal{P}\left(D_{E}\right) \backslash E$ (such an element exists since $\mathcal{I}_{R}\left(t_{R}\right)$ is the random graph). From $D_{E}$ and $I_{E}$ one can easily reconstruct the set $E$. More precisely, let $X$ be an element of $\mathcal{I}_{R}\left(t_{R}\right)$. Then $X$ belongs to $E$ if and only if $t_{R}$ models $\delta(X) \wedge X \subseteq$ $D_{E} \wedge \Psi\left(X, I_{E}\right)$.

Let now $G$ be a non-oriented finite graph. Since $\mathcal{I}_{R}\left(t_{R}\right)$ is the random graph, the graph $G$ appears as an induced subgraph of $\mathcal{I}_{R}\left(t_{R}\right)$ (cf. [Hod93]). Let $V$ be the set of vertices of $\mathcal{I}_{R}\left(t_{R}\right)$ inducing this subgraph.

For each subset $F$ of $V$, one can construct an element $v_{F}$ of $\mathcal{I}_{R}\left(t_{R}\right)$ which is connected to all the vertices in $F$ and to no vertex in $V \backslash F$. Knowing $V$, this element $v_{F}$ completely characterizes $F$. Let now $V^{\prime}$ be the set of all the $v_{F}$ 's for all subsets $F$ of $V$.

Let $t_{G}$ be the tree $t_{R}$ extended with markings describing $D_{V}, I_{V}, D_{V^{\prime}}$ and $I_{V^{\prime}}$. Using the trick mentioned above, we can define the formula $X \in V$ (similarly $X \in V^{\prime}$ ) to be $\delta(X) \wedge X \subseteq$ $D_{V} \wedge \Psi\left(X, I_{V}\right)$. We now want to finite sets interpret $\mathcal{P}^{W}(G)$ in $t_{G}$. Obviously, we can identify the elements of $\mathcal{P}^{W}(G)$ with the elements of $V^{\prime}$. Pursuing this idea, we define the interpretation $\mathcal{I}^{\prime}=\left(\delta^{\prime}(X), \Psi^{\prime}(X, Y), \Phi_{\subseteq}(X, Y)\right)$ in the following way. The universe is defined by $\delta^{\prime}(X)=X \in V^{\prime}$. The subset relation is defined by:

$$
\Phi_{\subseteq}(X, Y)=\forall Z \in V . \Psi(Z, X) \rightarrow \Psi(Z, Y)
$$

Finally the edge relation is defined by:

$$
\begin{aligned}
\Psi^{\prime}(X, Y) & =\operatorname{Singleton}(X) \wedge \text { Singleton }(Y) \\
& \wedge \exists X^{\prime}, Y^{\prime} \in V . \Psi\left(X^{\prime}, X\right) \wedge \Psi\left(Y^{\prime}, Y\right) \wedge\left(\Psi\left(X^{\prime}, Y^{\prime}\right)\right)
\end{aligned}
$$

where $\operatorname{Singleton}(Z)$ stands for $Z \in V^{\prime} \wedge \exists ! Y \in V . \Psi(Y, Z)$ and $\exists$ ! abbreviates "there exists one and only one".

Using the properties linking $V$ and $V^{\prime}$, it is not difficult to see that $\mathcal{I}^{\prime}\left(t_{G}\right)$ is (up to isomorphism) $\mathcal{P}^{W}(G)$. Furthermore, $\mathcal{I}^{\prime}$ does not depend on $G$. This finishes the proof of the claim and hence the proof of the theorem.

\footnotetext{
${ }^{1}$ To be precise the result in Cou97, applies to classes of finite graphs, whereas our trees $t_{G}$ may be infinite. But given an interpretation that produces a class of finite graphs from a class of infinite trees one can modify the interpretation such that one obtains the same resulting class of graphs from a class of finite trees.
} 
5.4. Intrinsic definability. Our last application of Theorem 4.3 concerns "intrinsic definability" of relations. This notion is the natural adaption of the notion of intrinsic regularity for automatic structures [KRS04. An automatic structure may have (up to isomorphism) several different presentations. These presentations can have different properties in the following sense: It might be possible to add a relation to the structure that is regular in one presentation but is not regular in the other presentation.

Consider, for example, the structure $\Delta_{1}$, i.e., the natural numbers with the successor relation. One automatic presentation is to use binary encodings of the numbers but it is also possible to use unary encoding. If we now add the predicate "being a power of 2", i.e., the set $\left\{2^{n} \mid n \in \mathbb{N}\right\}$, then this predicate is certainly not regular for the unary encoding but it is in the binary encoding (it corresponds to the set of all words of the form $10^{*}$ ). This means that this predicate is not intrinsically regular for $\Delta_{1}$ because it is regular in some presentation but not in all.

Accordingly, a relation is called intrinsically regular for a structure if it is regular in all automatic presentation of the structure. In [KRS04 this notion is studied and the question of a logical characterization of intrinsically regular relations is raised.

In [Bár06] it is shown that for the structure $\left(\{0,1\}^{*}, S_{0}, S_{1}, \sqsubseteq\right.$, el) (recall Example 2.3 above) each relation is either intrinsically regular or intrinsically non-regular, i.e., either it is regular in every presentation or non-regular in every presentation of the structure. Such a result can be used as a tool to show that certain structures are not automatic, which is a difficult task (cf. [BG00]). If we add a relation to the above structure and show that it is not regular in the natural automatic presentation, then we know that the structure extended by this relation has no automatic presentation at all.

In this subsection we show a stronger result for another structure. In terms of automatic structures we show that for $\mathcal{P}^{W}\left(\Delta_{1}\right)$ each relation is intrinsically regular or intrinsically non-regular for every tree-automatic presentation of $\mathcal{P}^{W}\left(\Delta_{1}\right)$.

For this, we adapt the notions to our setting. That is, intrinsic definability considers relations that are definable in every possible presentation of a structure by a finite sets interpretation from a fixed tree $t$. If we, for example, fix this tree to be $\Delta_{1}$, then this corresponds to intrinsic regularity for automatic structures.

Note that, in contrast to the previous sections, we now explicitly consider the presentations of elements of a structure, i.e., we distinguish different codings of the same structure.

Definition 5.5. Given a structure $\mathcal{T}$, a $\mathcal{T}$-presentation of a structure $\mathcal{S}$ of universe $\mathcal{U}$ is an injection $f$ from $\mathcal{U}$ to the finite subsets of $\mathcal{T}$ such that the set $f(\mathcal{U})$ as well as the image by $f$ of each relation $R$ of $\mathcal{S}$ are WMSO-definable on $\mathcal{T}$. That is, there is a formula $\phi_{\mathcal{U}}^{f}(X)$ over $\mathcal{T}$ defining the image of $\mathcal{U}$ under $f$, and for each relation $R$ of $\mathcal{S}$ there is a formula $\phi_{R}^{f}\left(X_{1}, \ldots, X_{r}\right)$ using the signature of $\mathcal{T}$ such that for all $u_{1}, \ldots, u_{r} \in \mathcal{U}$,

$$
\left(u_{1}, \ldots, u_{r}\right) \in R \quad \text { iff } \quad \mathcal{T} \models \phi_{R}^{f}\left(f\left(u_{1}\right), \ldots, f\left(u_{r}\right)\right),
$$

where $r$ denotes the arity of $R$.

Given such a $\mathcal{T}$-presentation $f$ of $\mathcal{S}$, it might be possible to add relations to $\mathcal{S}$ such that $f$ is still a $\mathcal{T}$-presentation of this extended structure. Such relations are called definable in $f$, i.e., $R^{\prime}$ is called definable in $f$ if $f$ is a $\mathcal{T}$-presentation of $\mathcal{S}$ extended by the relation $R^{\prime}$.

Note that to a $\mathcal{T}$-presentation $f$ of a structure $\mathcal{S}$ we can directly associate a finite sets interpretation $\mathcal{I}_{f}$ sending $\mathcal{T}$ to $\mathcal{S}$ up to isomorphism (the isomorphism being $f$ ). An additional relation $R^{\prime}$ is definable in $f$ if we can add to $\mathcal{I}_{f}$ a formula defining $R^{\prime}$. 
If $\mathcal{S}$ is the weak powerset structure $\mathcal{P}^{W}(\mathcal{T})$ of $\mathcal{T}$, then there is a canonical $\mathcal{T}$-presentation given by the identity mapping. We refer to this $\mathcal{T}$-presentation as the standard presentation of $\mathcal{P}^{W}(\mathcal{T})$.

The following lemma states that the "intrinsically definable" relations of $\mathcal{P}^{W}(t)$ for a tree $t$ are exactly those that are regular in the standard presentation of $\mathcal{P}^{W}(t)$.

Lemma 5.6. Let $t$ be a tree and $R$ be a relation over $\mathcal{P}^{W}(t)$. Then $R$ is definable in the standard presentation of $\mathcal{P}^{W}(t)$ iff $R$ is definable in all $t^{\prime}$-presentations $f$ of $\mathcal{P}^{W}(t)$ for all trees $t^{\prime}$.

Proof. Obviously, if $R$ is definable in all $t^{\prime}$-presentations $f$ of $\mathcal{P}^{W}(t)$ for all trees $t^{\prime}$, then $R$ is in particular definable in the standard presentation.

For the other direction, let $R$ be a relation of arity $r$ that is definable in the standard presentation of $\mathcal{P}^{W}(t)$ and let $\Phi_{R}\left(X_{1}, \ldots, X_{r}\right)$ be the defining formula, i.e., $\Phi_{R}$ is a formula over the signature of $t$ such that $t \models \Phi_{R}\left(U_{1}, \ldots, U_{r}\right)$ iff $\left(U_{1}, \ldots, U_{r}\right) \in R$ for all $U_{1}, \ldots, U_{r} \subseteq \operatorname{dom}(t)$. According to Proposition 2.7 we can construct an FO-interpretation $\mathcal{I}_{1}$ such that $\mathcal{I}_{1}\left(\mathcal{P}^{W}(t)\right)$ is the structure $\mathcal{P}^{W}(t)$ augmented with the relation $R$.

Let now $f$ be a $t^{\prime}$-presentation of $\mathcal{P}^{W}(t)$ and let $\mathcal{I}_{f}$ be the finite sets interpretation with $\mathcal{I}_{f}\left(t^{\prime}\right)=\mathcal{P}^{W}(t)$. Then $\left(\mathcal{I}_{1} \circ \mathcal{I}_{f}\right)\left(t^{\prime}\right)=\mathcal{I}_{1}\left(\mathcal{P}^{W}(t)\right)$, witnessing the definability of $R$ in the $t^{\prime}$-presentation $f$ of $\mathcal{P}^{W}(t)$.

The dual of Lemma 5.6, where definable is replaced by not definable, is not true in general. This is already the case for instance for $t=t^{\prime}=\Delta_{2}$, i.e., there is a relation $R$ which is not definable in the standard presentation of $\mathcal{P}^{W}\left(\Delta_{2}\right)$ but is definable in some $\Delta_{2}$-presentation of $\mathcal{P}^{W}\left(\Delta_{2}\right)$.

Consider, for example, the relation $R(x, y)$ that holds if $x$ is on the leftmost branch, $y$ is on the rightmost branch, and $x$ and $y$ are on the same level. It is not difficult to see that this relation is not WMSO-definable in $\Delta_{2}$. Hence, if we transfer $R$ to singletons on $\mathcal{P}^{W}\left(\Delta_{2}\right)$, it is not definable in the standard presentation of $\mathcal{P}^{W}\left(\Delta_{2}\right)$.

On the other hand, one can find a WMSO-interpretation $\mathcal{I}_{2}$ with $\mathcal{I}_{2}\left(\Delta_{2}\right) \sim\left(\Delta_{2}, R\right)$. The finite sets interpretation $\mathcal{P}^{W} \circ \mathcal{I}_{2}$ defines a $\Delta_{2}$-presentation of $\mathcal{P}^{W}\left(\Delta_{2}\right)$ in which $R$ (transferred to singletons) is regular. The construction of $\mathcal{I}_{2}$ is not very difficult. It suffices to redefine $\Delta_{2}$ inside itself such that the corresponding vertices of the leftmost and the rightmost branch are located close to each other, as for example done by the following mapping (where $w$ is any non-empty word over $\{0,1\}$ ):

$$
0^{n} \mapsto 0^{n}, \quad 1^{n} \mapsto 0^{n} 1, \quad 0^{n} 1 w \mapsto 0^{n} 11 w, \quad 1^{n} 0 w \mapsto 0^{n} 10 w .
$$

The reader can verify that the two successor relations and the relation $R$ are WMSOdefinable on this coding of $\Delta_{2}$.

However, in the particular case of $t=\Delta_{1}$ and $t^{\prime}=\Delta_{2}$ such a converse of Lemma 5.6 does hold as expressed in the following theorem.

Theorem 5.7. If $R$ is definable in some $\Delta_{2}$-presentation $f$ of $\mathcal{P}^{W}\left(\Delta_{1}\right)$, it is definable in every $\Delta_{2}$-presentation of $\mathcal{P}^{W}\left(\Delta_{1}\right)$.

As we can expect, by application of Theorem 4.3, we will obtain a WMSO-interpretation sending $\Delta_{2}$ to $\Delta_{1}$. The two following lemmas study this kind of interpretations and how they preserve definability. 
Lemma 5.8. Let $\mathcal{I}_{2}$ be a WMSO-interpretation sending $\Delta_{2}$ to $\Delta_{1}$ and $f_{2}$ be the injection from $\mathbb{N}$ to $\{0,1\}^{*}$ witnessing the isomorphism. Then there exists naturals $m, n>0$ and words $u, v, w_{0}, \ldots, w_{n-1} \in\{0,1\}^{*}$ such that for all naturals $k \geq 0$ and $p \in\{0, \ldots, n-1\}$,

$$
f_{2}(m+k n+p)=u v^{k} w_{p} .
$$

Proof. The general idea behind the proof is that the elements from the image of $f_{2}$ cannot be spread arbitrarily in $\Delta_{2}$ because the successor relation from $\Delta_{1}$ has to be definable in WMSO and hence must be recognizable by an automaton.

We denote by $U \subseteq\{0,1\}^{*}$ the image of $f_{2}$ and by $V$ the closure of $U$ by prefix. The set $V$ defines a subtree of $\Delta_{2}$. We now augment $V$ by markings containing information on the successor relation in such a way that these markings are definable in WMSO. Hence, the marked tree is regular, i.e., it has only finitely many non-isomorphic subtrees (see [Tho97] for more information on regular trees). From this regular tree we can define the words $u, v, w_{0}, \ldots, w_{n-1}$.

Let $\Phi_{\text {succ }}(x, y)$ be the formula of $\mathcal{I}$ that defines the successor relation succ of $\Delta_{1}$, and let $\mathcal{A}_{\text {succ }}$ be the equivalent tree automaton.

As succ is deterministic, one can easily show that $V$ contains only one infinite branch $B$. Otherwise, the relation succ has to jump infinitely often between two infinite branches leading to a contradiction as $\mathcal{A}_{\text {succ }}$ would also accept pairs of nodes that are not in succ. The argument is the same as in the proof of Proposition 5.1 where it is shown that $(\mathbb{N},+)$ is not WMSO-interpretable in any tree $t$. Furthermore, this branch $B$ is WMSO-definable.

Consider two nodes $x, y \in U$ such that $\Phi_{\text {succ }}(x, y)$ is satisfied, i.e., $f_{2}^{-1}(y)$ is the successor of $f_{2}^{-1}(x)$. We can describe how to get from $x$ to $y$ by a pair of words $\left(z_{x}, z_{x}^{\prime}\right)$ over $\{0,1\}$ meaning that $x=x^{\prime} z_{x}$ and $y=x^{\prime} z_{x}^{\prime}$ for the greatest common ancestor $x^{\prime}$ of $x$ and $y$. Again, using the determinism of succ one can show that the length of these words $z_{x}$, $z_{x}^{\prime}$ is bounded by some constant derived from the size of $\mathcal{A}_{\text {succ }}$. Hence, we can mark the vertices from $U$ by this information (using sets $X_{z, z^{\prime}}$ with $x \in X_{z, z^{\prime}}$ iff $\left(z_{x}, z_{x}^{\prime}\right)=\left(z, z^{\prime}\right)$ ). Obviously, this marking is WMSO-definable.

The last information that we attach to $V$ is for each node $x \in B$ the word $b_{x} \in\{0,1\}^{*}$ such that the node $x b_{x}$ is the smallest node in $U$ (smallest referring to the position in $\Delta_{1}$ ) such that all nodes bigger than $x b_{x}$ are below $x$, i.e., for all $y \in U$, if $f_{2}^{-1}\left(x b_{x}\right)<f_{2}^{-1}(y)$, then $x \sqsubseteq y$. The length of these $b_{x}$ is bounded because the relation that associates to each $x$ the node $b_{x}$ is WMSO-definable and deterministic. Hence, the marking of the nodes in $B$ by using sets $X_{b}$ with $x \in X_{b}$ iff $b_{x}=b$ is WMSO-definable.

The resulting tree $t$, consisting of the nodes in $V$ with the markings described above is WMSO-definable and hence regular. Let $u, v \in\{0,1\}^{*}$ such that $u, u v \in B$ and the subtrees of $t$ rooted at $u$ and $u v$ are isomorphic. Let $m=f_{2}^{-1}\left(u b_{u}\right), m^{\prime}=f_{2}^{-1}\left(u v b_{u v}\right)$, and define $n=m^{\prime}-m$. For $p \in\{0, \ldots, n-1\}$ let $w_{p} \in\{0,1\}^{*}$ be such that $f_{2}(m+p)=u w_{p}$. By the choice of $m$, such a $w_{p}$ always exists. In particular, $w_{0}=b_{u}$.

By the choice of $v$, we know that $f_{2}(m+k n)=u v^{k} w_{0}$ for all $k \geq 0$. Furthermore, as $t$ is marked by the information on how to get from one node in $U$ to its successor, we know that the ways to get from $f_{2}(m+k n+p)$ to $f_{2}(m+k n+p+1)$ are the same for all $k$. Hence, $f_{2}(m+k n+p)=u v^{k} w_{p}$.

Lemma 5.9. Let $\mathcal{I}_{2}$ be a WMSO-interpretation sending $\Delta_{2}$ to $\Delta_{1}$ and $f_{2}$ be the injection from $\mathbb{N}$ to $\{0,1\}^{*}$ witnessing the isomorphism. If $R$ is a relation over finite subsets of $f_{2}(\mathbb{N})$ $W M S O$-definable in $\Delta_{2}$, then its inverse image under $f_{2}$ is WMSO-definable in $\Delta_{1}$. 


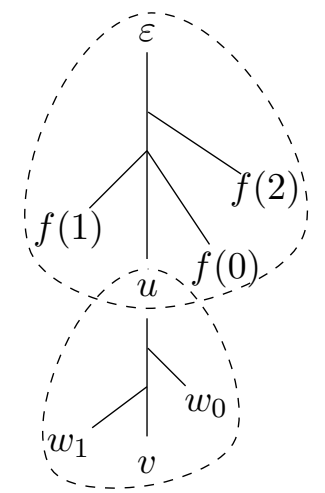

Figure 2: Regular subtree of $\Delta_{2}$ induced by the domain of the interpretation $\mathcal{I}_{2}$ from Lemmas 5.9 and 5.8. The part from $u$ to $v$ is iterated.

Proof. The formula $\Psi_{R}\left(X_{1}, \ldots, X_{r}\right)$ defining $R$ can be represented by a tree automaton $\mathcal{A}_{R}$ with state set $Q_{R}$. Using Lemma 5.8, this automaton can be simulated by an automaton $\mathcal{A}_{R}^{\prime}$ on $\Delta_{1}$ as we show in the following. On $\Delta_{1}$ automata and WMSO have the same expressive power and hence the construction of $\mathcal{A}_{R}^{\prime}$ suffices to prove the lemma.

Let $u, v, w_{0}, \ldots, w_{n-1}$ be as in Lemma 5.8. The states of $\mathcal{A}_{R}^{\prime}$ correspond to partial runs of $\mathcal{A}_{R}$ on a finite subtree of $\Delta_{2}$ that

- is rooted at $\epsilon$ and induced by the elements $f_{2}(0), \ldots, f_{2}(m-1)$ for the first segment of $\Delta_{1}$

- rooted at $u v^{k}$ and induced by the words $v, w_{0}, \ldots, w_{n-1}$ for the following segments of $\Delta_{1}$.

The corresponding parts of $\Delta_{2}$ on which $\mathcal{A}_{R}^{\prime}$ has to simulate a run of $\mathcal{A}_{R}$ are depicted in Figure 2 (for $n=2$ and $m=3$ ).

For the formal definitions, let $U_{0}$ be the set of all nodes that are prefix of $u$ or of some $f_{2}(i)$ for $0 \leq i<m$, and let $U_{1}$ be the set of all nodes that are prefix of $v$ or one of $w_{0}, \ldots, w_{n-1}$. The set $U_{0}$ corresponds to the upper finite tree in Figure 2 surrounded by a dashed line, and the set $U_{1}$ to the finite tree rooted at $u$.

The automaton $\mathcal{A}_{R}^{\prime}$ reads a word $\alpha \in\left(\{0,1\}^{r}\right)^{\omega}$ and has to decide if this labeling transferred by $f_{2}$ to $\Delta_{2}$ corresponds to a tuple of sets in $R$. To simulate a run of $\mathcal{A}_{R}$ it guesses partial runs, starting with a partial run on $U_{0}$, and then continuing with $U_{1}$, the periodic part of the tree.

More formally, it starts by guessing a pair $\left(\rho_{0}, \lambda_{0}\right)$ with mappings $\rho_{0}: U_{0} \rightarrow Q_{R}$ and $\lambda_{0}:\left\{f_{2}(0), \ldots, f_{2}(m-1)\right\} \rightarrow\{0,1\}^{r}$ such that $\rho_{0}$ corresponds to a partial run of $\mathcal{A}_{R}$ on $U_{0}$ with labels corresponding to $\lambda_{0}$. In the next steps, $\mathcal{A}_{R}^{\prime}$ verifies if the guessed labeling is correct, i.e., if $\alpha(i)=\lambda_{0}\left(f_{2}(i)\right)$. When reaching position $m, \mathcal{A}_{R}^{\prime}$ guesses a new pair $\left(\rho_{1}, \lambda_{1}\right)$, now with mappings $\rho_{1}: U_{1} \rightarrow Q_{R}$ and $\lambda_{1}:\left\{w_{0}, \ldots, w_{n-1}\right\} \rightarrow\{0,1\}^{r}$ such that $\rho_{1}$ is a possible continuation of $\rho_{0}$ on the subtree rooted at $u$ that is shown in Figure 2 and labeled according to $\lambda_{1}$. The guessed labeling is again verified on the segment $m, \ldots, m+n-1$ and then a pair $\left(\rho_{2}, \lambda_{2}\right)$ of the same type as $\left(\rho_{1}, \lambda_{1}\right)$ is guessed, and so on. The automaton accepts if the concatenation of the guessed partial runs on the path $u v^{\omega}$ satisfies the acceptance condition of $\mathcal{A}_{R}$. For this to work, the guessed partial runs have to be such that they can 
be continued to accepting runs on the "blank parts" of $\Delta_{2}$, i.e., those infinite subtrees that do not contain a node from the image of $f_{2}$.

Using this Lemma we can prove Theorem 5.7 .

Proof of Theorem 5.7. Assuming a $\Delta_{2}$-presentation $f$ of $\mathcal{P}^{W}\left(\Delta_{1}\right)$ and $\mathcal{I}$ the corresponding finite sets interpretation, one obtains by Corollary 4.4 a WMSO-interpretation $\mathcal{I}_{2}$ with $\mathcal{I}_{2}\left(\Delta_{2}\right)=\Delta_{1}$. Let $f_{2}: \mathbb{N} \rightarrow\{0,1\}^{*}$ be the injection witnessing this isomorphism and let $\tilde{f}_{2}$ be its extension to sets.

We now have two ways to obtain isomorphic copies of $\mathcal{P}^{W}\left(\Delta_{1}\right)$ from $\Delta_{2}$ : by applying $\mathcal{I}$ and by applying $\mathcal{I}_{2}$ followed by $\mathcal{P}^{W}$. These two ways yield isomorphic structures and hence there is an isomorphism $h$ sending $\mathcal{I}\left(\Delta_{2}\right)$ to $\mathcal{P}^{W}\left(\mathcal{I}_{2}\left(\Delta_{2}\right)\right)$. We obtain the following picture, where dashed arrows represent interpretations, while normal arrows are for isomorphisms:

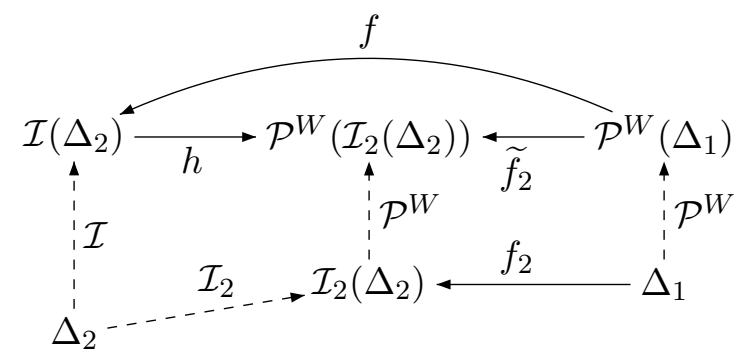

We show that we can define the isomorphism $h$ on $\Delta_{2}$ by a WMSO-formula. To understand this, consider a finite set $U$ of naturals, i.e., an element of $\mathcal{P}^{W}\left(\Delta_{1}\right)$. This set $U$ corresponds to two sets $X$ and $Y$ of nodes of $\Delta_{2}$, namely $X=f(U)$ and $Y=\tilde{f}_{2}(U)$. The isomorphism $h$ relates these two sets, i.e., $h(X)=Y$. This relation can be defined in WMSO using the formula Code that we obtain from Theorem 4.3 and that is used to construct $\mathcal{I}_{2}$ in Corollary 4.4. The formula $\operatorname{Code}(X, x)$ relates each subset of $\Delta_{2}$ that is an atom in $\mathcal{P}^{W}\left(\Delta_{1}\right)=\mathcal{I}\left(\Delta_{2}\right)$ to a single node $x$ of $\Delta_{2}$. Assume that the set $X$ represents the atom $\{n\}$. Then the unique $x$ such that $\operatorname{Code}(X, x)$ is satisfied represents $n$ in $\mathcal{I}_{2}\left(\Delta_{2}\right)=\Delta_{1}$ (by the construction of $\mathcal{I}_{2}$ in the proof of Corollary 4.4), and the singleton $\{x\}$ represents the atom $\{n\}$ in $\mathcal{P}^{W}\left(\mathcal{I}_{2}\left(\Delta_{2}\right)\right)$. We get that $h(X)=x$, i.e., the formula Code defines the isomorphism $h$ on the level of atoms. It is easy to extend this to sets:

$$
\phi_{h}(X, Y)=\forall y\left(y \in Y \leftrightarrow \exists Z\left(\Phi_{\subseteq}(Z, X) \wedge \operatorname{Code}(Z, y)\right)\right) .
$$

Let now $R$ be a relation over $\mathcal{P}^{W}\left(\Delta_{1}\right)$ which is definable in $f$. This means that $f(R)$ is WMSO-definable in $\Delta_{2}$. Since $h$ is WMSO-definable, it follows that $h(f(R))$ is also WMSO-definable in $\Delta_{2}$. Finally, by Lemma 5.9 we obtain that $\tilde{f}_{2}^{-1}(h(f(R)))$ is WMSOdefinable in $\Delta_{1}$. Since $\tilde{f}_{2}^{-1} \circ h \circ f$ is obtained as a composition of isomorphisms, it is an automorphism of $\mathcal{P}^{W}\left(\Delta_{1}\right)$. Remark now that the identity is the only automorphism of $\mathcal{P}^{W}\left(\Delta_{1}\right)$ (a property inherited from $\Delta_{1}$ ). It follows that $\tilde{f}_{2}{ }^{-1}(h(f(R)))$ equals $R$. And consequently $R$ is definable in the standard presentation. By Lemma 5.6] it is definable in every presentation. 


\section{Proof of the Main Result}

The proof of Theorem 4.3 is rather complex and split into several parts. In Subsection 6.1. we introduce the key notions used afterwards while we make the scheme of the proof more precise. This will also be the occasion for explaining the content of Subsections 6.2. 6.3, 6.4, 6.5. In Subsection 6.6, things are put together and the proof of Theorem 4.3 is finally given.

6.1. First definitions and presentation of the proof. We assume from now that a finite sets interpretation $\mathcal{I}=\left(\delta(X), \phi_{\preceq}(X, Y)\right)$ is fixed. Along the whole proof we use a tree $t$ together with a set $E$ and the isomorphism $f$ that are assumed to satisfy the equality

$$
f\left(\mathcal{P}^{F}(E)\right)=\mathcal{I}(t) .
$$

The reader must keep in mind that none of the constructions we perform makes use of $t$, $E$, or $f$. Hence, the result will hold for any such tree, set, and isomorphism. This lightens the presentation of the proof by avoiding to systematically quantify over those objects.

We consider the set Atoms of finite subsets of $t$ representing atoms of the powerset lattice, i.e.,

$$
\text { Atoms }=\{f(\{u\}): u \in E\} .
$$

The set Atoms can be defined as the set of finite subsets of $t$ which are minimal - for the $\phi_{\preceq}$ formula seen as an ordering — and distinct from the minimal element itself (which is $f(\emptyset)$ ). This description can be done in weak monadic second-order logic. Hence Atoms is regular in $t$ and there exists an automaton

$$
\mathcal{A}_{\text {Atoms }}=\left(Q_{\text {Atoms }}, q_{\text {Atoms }}^{\text {in }}, \delta_{\text {Atoms }}, \Omega_{\text {Atoms }}\right)
$$

accepting the language Atoms. We also consider the binary relation $M e m$ over $\mathcal{I}(t)$ defined as the image under $f$ of the $\in$ relation in $\mathcal{P}^{F}(E)$, i.e.,

$$
M e m=\{(f(\{u\}), f(V)): u \in V \subseteq E \text { and } V \text { finite }\} .
$$

This $M e m$ relation is also definable in weak monadic second-order logic, and consequently is regular. We fix

$$
\mathcal{A}_{\mathrm{Mem}}=\left(Q_{\mathrm{Mem}}, q_{\mathrm{Mem}}^{\mathrm{in}}, \delta_{\mathrm{Mem}}, \Omega_{\mathrm{Mem}}\right)
$$

to be an automaton recognizing the relation $\mathrm{Mem}$.

Recall that the theorem we want to prove claims the existence of a formula $\operatorname{Code}(X, x)$ such that the corresponding relation is an injection from Atoms into dom $(t)$.

Our goal in the construction of Code is to uniquely attach to each $X$ in Atoms an element in $\operatorname{dom}(t)$ in a WMSO-definable way. As a first approximation, in Subsections 6.2. 6.3 and 6.4, we define a mapping Index which assigns to each $X$ in Atoms a node in dom(t). Though the Index mapping is not in general an injection from Atoms into dom $(t)$, it does not either concentrate a lot of indices in the same area of the tree $t$. Formally, if we set $D(x)$ for $x \in t$ to be the cardinality of Index ${ }^{-1}(x)$, then by Lemmas 6.6 and 6.12, $D$ happens to be a sparse distribution (see Definition 6.1 below). Subsection 6.5 is dedicated to the study of sparse distributions. The central lemma of this part, Lemma 6.17, establishes that, given elements concentrated according to a sparse distribution, we can uniformly redistribute them in $\operatorname{dom}(t)$ in a unique WMSO-definable way. Applied to our case, this means that the Index mapping can be transformed into an injection by use of WMSO-formulas. And this last step is used in Subsection 6.6 for terminating the proof of Theorem 4.3. 
The key definition connecting the two main parts of the proof (definition of the Index mapping and turning it into an injection) is the notion of sparsity. This definition requires the notion of zone. A zone $Z$ in $t$ is a connected - where $t$ is seen as an non-oriented graph - subset of $d o m(t)$. That is, $Z$ contains a minimal element w.r.t. to the prefix ordering, and whenever $x \sqsubseteq y \sqsubseteq z$ for $x, z$ in $Z$, then also $y \in Z$. A zone $Z$ is completely characterized by its least element $x$, and by the minimal elements $x_{1}, \ldots, x_{n}$ that are below $x$ and not in $Z$. The elements $\left\{x, x_{1}, \ldots, x_{n}\right\}$ are called the frontier of the zone. Given nodes $x, x_{1}, \ldots, x_{n}$ of $t$ such that the $x_{i}$ are pairwise incomparable and $x \sqsubseteq x_{i}$ for all $i$, we define $N_{t}^{x, x_{1}, \ldots, x_{n}}$ to be the set of nodes $y$ such that $x \sqsubseteq y$ and $x_{i} \nsubseteq y$ for all $i \in[n]$ where $[n]$ denotes the set $\{1, \ldots n\}$. By construction, $N_{t}^{x, x_{1}, \ldots, x_{n}}$ is the only zone which has frontier $\left\{x, x_{1}, \ldots, x_{n}\right\}$.

Definition 6.1. A distribution $D$ is a mapping from $\operatorname{dom}(t)$ to $\mathbb{N}$. For $Z$ a finite zone, $D(Z)$ stands for $\sum_{x \in Z} D(x)$. A distribution $D$ is $K$-sparse for some $K \in \mathbb{N}$ if for every finite zone $Z$ of frontier $F, D(Z) \leq|Z|+K|F|$. A distribution is strongly $K$-sparse if for any finite zone $Z$ of frontier $F, D(Z) \leq K|F|$.

Sparsity tells us that no finite zone contains more indices than its size plus a factor linearly depending on the size of the frontier.

6.2. Important nodes. In order to construct the mapping Index, given an element $X$ of Atoms, we first define the set $I(X) \subseteq \operatorname{dom}(t)$ of its important nodes via combinatorial constraints. Essentially, we try to locate the places where "important coding decisions" are made by the automaton $\mathcal{A}_{\text {Atoms }}$ when reading $X$. In the present Subsection, we provide the key combinatorial lemmas concerning important nodes.

Then, depending on the shape of the set $I(X)$ two cases are separated and two distinct definitions of Index are given. The first kind of index is called standard index - noted $\operatorname{SIndex}(X)-$ and is the subject of Subsection 6.3. The other kind is called branch index - noted BIndex $(X)$ - and is the subject of Subsection 6.4.

Let us first introduce a convenient notation for studying the behavior of the automata $\mathcal{A}_{\text {Atoms }}$ and $\mathcal{A}_{\text {Mem }}$ over zones: For a zone $Z=N_{t}^{x, x_{1}, \ldots, x_{n}}$ and states $q, q_{1}, \ldots, q_{n} \in Q_{\text {Atoms }}$, we denote by $\operatorname{Atoms}\left(q, Z, q_{1}, \ldots, q_{n}\right)$ the set of all $X \subseteq Z$ such that there exists $X^{\prime} \subseteq \operatorname{dom}(t)$ with

- $X=X^{\prime} \cap Z$, and

- $X^{\prime}$ is accepted by $\mathcal{A}_{\text {Atoms }}$ with a run $\rho$ such that $\rho(x)=q$ and $\rho\left(x_{i}\right)=q_{i}$ for all $i \in[n]$.

Similarly, for $q, q_{1}, \ldots, q_{n} \in Q_{M e m}$ we denote by $\operatorname{Mem}\left(q, Z, q_{1}, \ldots, q_{n}\right)$ the set of all pairs $(X, Y)$ with $X, Y \subseteq Z$ such that there are $X^{\prime}, Y^{\prime} \subseteq \operatorname{dom}(t)$ with

- $X=X^{\prime} \cap Z, Y=Y^{\prime} \cap Z$, and

- $\left(X^{\prime}, Y^{\prime}\right)$ is accepted by $\mathcal{A}_{M e m}$ with a run $\rho$ such that $\rho(x)=q$ and $\rho\left(x_{i}\right)=q_{i}$ for all $i \in[n]$.

The definition of important nodes is then the following, where the constant $K_{\text {im }}$ is chosen to make the combinatorial arguments in the subsequent lemmas work.

Definition 6.2. Let $K_{\mathrm{im}}=\left(2\left|Q_{M e m}\right|+1\right)\left|Q_{\text {Atoms }}\right|$. Given $X \in$ Atoms, a node $x \in \operatorname{dom}(t)$ is called important for $X$ if

$$
\mid\left\{Y \subseteq N_{t}^{x}:\left(X-N_{t}^{x}\right) \cup Y \in \text { Atoms }\right\} \mid>K_{\text {im }} .
$$

We denote by $I(X)$ the set of important nodes for $X$. 
Hence a node $x$ is important for $X$ if there are many - i.e., more than $K_{\mathrm{im}}$ - ways to modify $X$ below $x$ while remaining in Atoms. Intuitively, without knowing how $X$ looks like below $x$, we cannot say much about which atom is coded because there are too many possibilities left. Remark that the set $I(X)$ is by definition prefix closed. The fundamental property that we show in Lemma 6.4 is that for an important node $x$ of $X$, the part of $X$ that is not below $x$ comes from a set of small size. To prove this lemma we need its combinatorial core stated in the following lemma.

Lemma 6.3. Let $K_{\mathrm{c}}=2\left|Q_{\text {Mem }}\right|+1$. For any two disjoint zones $Z$ and $Z^{\prime}$ of respective frontiers $F=\left\{x, x_{1}, \ldots, x_{n}\right\}$ and $F^{\prime}=\left\{x^{\prime}, x_{1}^{\prime}, \ldots, x_{m}^{\prime}\right\}$, and all accepting runs $\rho$ of $\mathcal{A}_{\text {Atoms }}$

$$
\begin{aligned}
\text { either } & \mid \text { Atoms }\left(\rho(x), Z, \rho\left(x_{1}\right), \ldots, \rho\left(x_{n}\right)\right) \mid<K_{\mathrm{c}}, \\
\text { or } & \mid \text { Atoms }\left(\rho\left(x^{\prime}\right), Z^{\prime}, \rho\left(x_{1}^{\prime}\right), \ldots, \rho\left(x_{m}^{\prime}\right)\right) \mid<K_{\mathrm{c}} .
\end{aligned}
$$

Proof. It is sufficient for us to prove the result for two complementary zones. This comes from the fact that increasing a zone also increases the number of possible projections w.r.t. a fixed run, i.e., $\left|\operatorname{Atoms}\left(\rho(x), Z, \rho\left(x_{1}\right), \ldots, \rho\left(x_{n}\right)\right)\right| \leq\left|\operatorname{Atoms}\left(\rho(y), Z^{\prime \prime}, \rho\left(y_{1}\right), \ldots, \rho\left(y_{\ell}\right)\right)\right|$ for a zone $Z^{\prime \prime}$ of frontier $\left\{y, y_{1}, \ldots, y_{\ell}\right\}$ with $Z \subseteq Z^{\prime \prime}$. Hence, we will assume $Z$ to be $N_{t}^{\epsilon, x}$ and $Z^{\prime}$ to be $N_{t}^{x}$ for some node $x$.

Assume that for some $K \geq K_{\mathrm{c}}$ we have distinct sets $X_{1}, \ldots, X_{K}$ in $\operatorname{Atoms}(\rho(\epsilon), Z, \rho(x))$ and distinct sets $X_{1}^{\prime}, \ldots, X_{K}^{\prime}$ in Atoms $\left(\rho(x), Z^{\prime}\right)$. Then, for every $i, j \in[K]$, let $Y_{i, j}$ be $X_{i} \cup$ $X_{j}^{\prime}$. As the union of $Z$ and $Z^{\prime}$ gives the whole domain of $t$, we have $Y_{i, j} \in$ Atoms for all $i, j \in[K]$.

Let us now consider the set Comb of possible combinations of the $Y_{i, j}$, combination in the sense of the relation $M e m$. More precisely, $A \subseteq d o m(t)$ is in $C o m b$ if whenever $(Y, A) \in M e m$ holds for some atom $Y$, then $Y=Y_{i, j}$ for some $i, j$. The cardinality of Comb is $2^{K^{2}}$. We now show by a combinatorial argument that $\mathcal{A}_{M e m}$ cannot distinguish all the elements from Comb because the amount of information that can be passed between the two zones $Z$ and $Z^{\prime}$ is limited by the number of states in $Q_{M e m}$.

For this purpose, we define for each $A \in C o m b, f_{A}:[K] \times Q_{M e m} \rightarrow\{0,1\}$ and $g_{A}$ : $Q_{\text {Mem }} \times[K] \rightarrow\{0,1\}$ by

$$
\begin{aligned}
& f_{A}(i, q)= \begin{cases}1 & \text { if }\left(X_{i}, A \cap Z\right) \in \operatorname{Mem}\left(q_{\text {Mem }}^{\text {in }}, Z, q\right), \\
0 & \text { else, }\end{cases} \\
& g_{A}(q, j)= \begin{cases}1 & \text { if }\left(X_{j}^{\prime}, A \cap Z^{\prime}\right) \in \operatorname{Mem}\left(q, Z^{\prime}\right), \\
0 & \text { else. }\end{cases}
\end{aligned}
$$

It is obvious that if two sets $A, B \in C o m b$ are such that $f_{A}=f_{B}$ and $g_{A}=g_{B}$, then $\left(Y_{i, j}, A\right) \in M e m$ iff $\left(Y_{i, j}, B\right) \in M e m$ for all $i, j \in[K]$. This means, by definition of Comb, that $A=B$.

However, there are only $2^{2\left|Q_{M e m}\right| K}$ different possible values for the pair $f_{A}, g_{A}$. Hence we obtain $|C o m b| \leq 2^{2\left|Q_{M e m}\right| K}$. This contradicts $|C o m b|=2^{K^{2}}$.

The following lemma shows that the possibilities to code an atom 'above' an important node are bounded.

Lemma 6.4. For each node $x$ we have $\mid\left\{X \cap N_{t}^{\epsilon, x}: X \in\right.$ Atoms and $\left.x \in I(X)\right\} \mid<K_{\mathrm{im}}$. 
Proof. We are aiming at a contradiction to Lemma 6.3 for $Z=N_{t}^{\epsilon, x}$ and $Z^{\prime}=N_{t}^{x}$.

For each $X$ with $x \in I(X)$ there are more than $K_{\text {im }}$ many $Y \subseteq N_{t}^{x}$ such that $X_{Y, x}:=$ $\left(X-N_{t}^{x}\right) \cup Y$ is in Atoms. Since $K_{\mathrm{im}}=\left|Q_{\text {Atoms }}\right| \cdot K_{\mathrm{c}}$ (with $K_{\mathrm{c}}$ from Lemma 6.3), we can choose a state $q_{X, x} \in Q_{\text {Atoms }}$ such that more than $K_{\mathrm{c}}$ of these $X_{Y, x}$ are accepted by $\mathcal{A}_{\text {Atoms }}$ with a run that labels $x$ with $q_{X, x}$. This means that $\mid$ Atoms $\left(q_{X, x}, N_{t}^{x}\right) \mid \geq K_{\mathrm{c}}$.

Now, assume that there are $K_{\mathrm{im}}$ different $X \in$ Atoms with $x \in I(X)$ that differ on $N_{t}^{\epsilon, x}$. Then there are at least $K_{\mathrm{c}}$ such sets $X_{1}, \ldots, X_{K_{\mathrm{c}}}$ with $q_{X_{1}, x}=\cdots=q_{X_{K_{\mathrm{c}}, x}}=: q$. In particular, we obtain $\mid$ Atoms $\left(q_{\text {Atoms }}^{\text {in }}, N_{t}^{x}, q\right) \mid \geq K_{\mathrm{c}}$. Together with $\left|\operatorname{Atoms}\left(q, N_{t}^{x}\right)\right| \geq K_{\mathrm{c}}$ from above we obtain the desired contradiction.

6.3. Standard indices. We now address the problem of computing Index $(X)$ for some atom $X$ under the assumption that $I(X)$ is not an infinite branch (the case when $I(X)$ is an infinite branch is treated in Subsection 6.4). Since we call this case the standard case, we will denote the index defined for such atoms $X$ by $\operatorname{SIndex}(X)$. The simplest case is that $I(X)$ is totally ordered by $\sqsubseteq$, i.e., $I(X)$ is a finite path starting from the root. We simply define $\operatorname{SIndex}(X)$ to be the last node on this path. The other case corresponds to $I(X)$ not being a finite path nor an infinite branch. This corresponds to $I(X)$ not being totally ordered by $\sqsubseteq$. In this situation, we define $\operatorname{SIndex}(X)$ to be the first node at which $I(X)$ splits into two paths. Those two cases are unified in the following definition.

Definition 6.5. For $X \in$ Atoms such that $I(X)$ is not an infinite branch, the index of $X$, written $\operatorname{SIndex}(X)$, is the maximal element in $I(X)$ which is comparable to every element in $I(X)$.

As already mentioned, the intention of this definition is that $S \operatorname{Index}(X)$ roughly locates in the tree where the main information concerning the atom coded by $X$ lies. This location is far from being precise, and many elements of Atoms may have the same index. However, we will see that it is possible to obtain a good understanding of the repartition of the standard indices. The following lemma gives precise bounds on the quantity of indices that may occur in a zone, i.e., it states that the distribution assigning to each node $x$ the number of $X$ such that $\operatorname{SIndex}(X)=x$ is sparse.

Lemma 6.6 (sparsity). There is a constant $K_{\mathrm{s}}$ such that $\left|\operatorname{SIndex}^{-1}(Z)\right| \leq|Z|+K_{\mathrm{s}}|F|$ for every finite zone $Z$ of frontier $F$.

Proof. Denote the elements of the frontier of $Z$ by $x$ and $x_{1}, \ldots, x_{n}$, i.e., $Z=N_{t}^{x, x_{1}, \ldots, x_{n}}$. The proof of the lemma consists of two steps. We first show that for atoms $X$ such that $\operatorname{SIndex}(X)$ is inside $Z$, the amount of information located outside $Z$ is bounded. More precisely, we first show for $M:=K_{\mathrm{im}} \cdot\left|Q_{\text {Atoms }}\right|$

(a) $\mid\left\{X \cap N_{t}^{\epsilon, x}: X \in\right.$ Atoms and $\left.\operatorname{SIndex}(X) \in Z\right\} \mid<M$ and

(b) $\mid\left\{X \cap N_{t}^{x_{i}}: X \in\right.$ Atoms and $\left.\operatorname{SIndex}(X) \in Z\right\} \mid<M$ for all $i \in[n]$.

For (a) note that from $\operatorname{SIndex}(X) \in Z$, the definition of SIndex, and the prefix closure of $I(X)$ we obtain that $x \in I(X)$. Therefore,

$$
\begin{array}{r}
\left\{X \cap N_{t}^{\epsilon, x}: X \in \text { Atoms and } \operatorname{SIndex}(X) \in Z\right\} \\
\subseteq\left\{X \cap N_{t}^{\epsilon, x}: X \in \text { Atoms and } x \in I(X)\right\}
\end{array}
$$

and (a) follows from Lemma 6.4. 
For (b) we show that for each $X \in \operatorname{SIndex}^{-1}(Z)$ and each $x_{i}$ there is a state $q \in Q_{\text {Atoms }}$ such that $X \cap N_{t}^{x_{i}} \in \operatorname{Atoms}\left(q, N_{t}^{x_{i}}\right)$ and $\left|\operatorname{Atoms}\left(q, N_{t}^{x_{i}}\right)\right|<K_{\text {im. }}$. From this, (b) follows because each $X \cap N_{t}^{x_{i}}$ comes from one of at most $\left|Q_{\text {Atoms }}\right|$ many sets of size less than $K_{\mathrm{im}}$. We distinguish two cases.

If $x_{i} \notin I(X)$, then we take $q$ to be the state at $x_{i}$ in an accepting run of $\mathcal{A}_{\text {Atoms }}$ on $X$. From the definition of $I(X)$ we immediately obtain the desired property.

Else, if $x_{i} \in I(X)$, by definition of standard indices there must some $y \in I(X)$ incomparable to $x_{i}$, the index of $X$ being the deepest common ancestor of $x_{i}$ and $y$. From the definition of important nodes for $X$ and from $K_{\mathrm{im}}=\left|Q_{\text {Atoms }}\right| \cdot K_{\mathrm{c}}$ we obtain that there exists a set $Y \subseteq N_{t}^{y}$ such that $\left(X-N_{t}^{y}\right) \cup Y$ is in Atoms and is accepted with a run of $\mathcal{A}_{\text {Atoms }}$ that labels $y$ by a state $q^{\prime}$ such that $\left|A \operatorname{toms}\left(q^{\prime}, N_{t}^{y}\right)\right| \geq K_{\mathrm{c}}$. Let $q$ be the state assumed at node $x_{i}$ by this run. From Lemma 6.3 applied to the zones $N_{t}^{x_{i}}, N_{t}^{y}$, and to the aforementioned run, we can conclude that $\left|\operatorname{Atoms}\left(q, N_{t}^{x_{i}}\right)\right|<K_{\mathrm{c}}$. The desired property follows from $K_{\mathrm{c}} \leq K_{\mathrm{im}}$. This finishes the proof of (b).

After these preliminary considerations, we come back to the claim of the lemma. We denote the elements from $\left\{X \cap N_{t}^{\epsilon, x}: \operatorname{SIn} \operatorname{dex}(X) \in Z\right\}$ by $X^{1}, \ldots, X^{M}$ and the elements from $\left\{X \cap N_{t}^{x_{i}}: \operatorname{SIndex}(X) \in Z\right\}$ by $X_{i}^{1}, \ldots, X_{i}^{M}$ (the same element can be represented more than once, what is important is that all elements are represented).

Now, consider the set $C o m b$ of all combinations of atoms from $\operatorname{SIndex}^{-1}(Z)$ (in the same sense as in the proof of Lemma 6.3). A combination $A \in C o m b$ is entirely characterized by the following objects

- the set $A \cap Z$,

- the mapping $f_{A, x}:[M] \times Q_{M e m} \rightarrow\{0,1\}$ with

$$
f_{A, x}(j, q)= \begin{cases}1 & \text { if }\left(X^{j}, A \cap N_{t}^{\epsilon, x}\right) \in \operatorname{Mem}\left(q_{M e m}^{\mathrm{in}}, N_{t}^{\epsilon, x}, q\right), \\ 0 & \text { else, }\end{cases}
$$

- and the mapping $f_{A, x_{i}}: Q_{M e m} \times[M] \rightarrow\{0,1\}$ with

$$
f_{A, x_{i}}(q, j)= \begin{cases}1 & \text { if }\left(X_{i}^{j}, A \cap N_{t}^{x_{i}}\right) \in \operatorname{Mem}\left(q, N_{t}^{x_{i}}\right), \\ 0 & \text { else. }\end{cases}
$$

Thus, $|C o m b| \leq 2^{|Z|} \cdot 2^{M\left|Q_{M e m}\right|} \cdot \prod_{i=1}^{n} 2^{\left|Q_{M e m}\right| M}$. For $K_{\mathrm{s}}=\left|Q_{M e m}\right| M$ we obtain

$$
2^{\mid \text {SIndex }^{-1}(Z) \mid}=\mid \text { Comb } \mid \leq 2^{|Z|+|F| K_{\mathrm{s}}}
$$

and hence $\left|\operatorname{SIndex}^{-1}(Z)\right| \leq|Z|+K_{\mathrm{s}}|F|$.

6.4. Treatment of infinite branches. It is possible that for some $X \in$ Atoms the set $I(X)$ of important nodes is an infinite branch. For these $X$ we also develop a notion of index, called BIndex $(X)$, and show that the distribution obtained in this way is strongly sparse. Since the sum of a $K$-sparse distribution and of a strongly $K^{\prime}$-sparse distribution is a $K+K^{\prime}$-sparse distribution, we can add the indices corresponding to infinite branches to the other indices without affecting the sparsity of the induced distribution.

In this subsection, we call the infinite branches that are equal to $I(X)$ for some atom $X$ important branches. We start with the helpful observation that the number of elements of Atoms corresponding to the same important branch is bounded.

Lemma 6.7. For every important branch $B,\left|I^{-1}(B)\right|<K_{\text {im }}$. 
Proof. If there are $K_{\mathrm{im}}$ different sets in $I^{-1}(B)$, then we can pick a node $x$ on $B$ such that all these sets differ on the zone $N_{t}^{\epsilon, x}$. Since $x$ is important for all $X$ in $I^{-1}(B)$, we obtain a contradiction to Lemma 6.4.

Our goal is to associate to every important branch $B$ a node $\operatorname{VInd}(B)$ on $B$ such that (1) at most $K_{\text {im }}$ branches are mapped to the same node by VInd, and

$(2)$ if some $\operatorname{VInd}\left(B^{\prime}\right)$ is above $\operatorname{VInd}(B)$, then $\operatorname{VInd}(B)$ is not in $B^{\prime}$.

Those two properties are established in Lemma 6.11. Then Lemma 6.12 uses those two properties for concluding that VInd $\circ I$ has a strongly sparse distribution.

Our main tool for constructing VInd is to produce a well-founded order for branches. For this, we define $R \operatorname{Ind}(B)$ for every important branch $B$ by

$$
R \operatorname{Ind}(B)=\min \left\{x \in B: \exists X \subseteq N_{t}^{\epsilon, x}, I(X)=B\right\} .
$$

Since we consider finite sets interpretations, $R \operatorname{Ind}(B)$ is always defined. Lemma 6.4 applied to the node $R \operatorname{Ind}(B)$ directly leads to the following lemma.

Lemma 6.8. For all nodes $x,\left|R \operatorname{Ind}^{-1}(x)\right|<K_{\mathrm{im}}$.

The well-foundedness argument announced above is then the following.

Lemma 6.9. For every important branch $B$, there are finitely many important branches $B^{\prime}$ such that $R \operatorname{Ind}\left(B^{\prime}\right) \sqsubseteq R \operatorname{Ind}(B)$.

Proof. One has that $R \operatorname{Ind}\left(B^{\prime}\right) \sqsubseteq R \operatorname{Ind}(B)$ iff $B^{\prime}$ belongs to $\cup_{x \sqsubseteq R \operatorname{Ind}(B)} R \operatorname{Ind}^{-1}(x)$. This set is finite by Lemma 6.8.

Now, we can define for a branch $B$ the index $\operatorname{VInd}(B)$ as being the first node in $B$ below $R \operatorname{Ind}(B)$ which is not lying on an important branch strictly inferior with respect to comparing the RInd values. Formally

$$
\operatorname{VInd}(B)=\min \left\{x \in B: R \operatorname{Rnd}(B) \sqsubseteq x, \forall B^{\prime} . R \operatorname{Rnd}\left(B^{\prime}\right) \sqsubset R \operatorname{Rnd}(B) \rightarrow x \notin B^{\prime}\right\} .
$$

This definition is sound thanks to Lemma 6.9. Furthermore, VInd and RInd can be related in the following way.

Lemma 6.10. $\operatorname{VInd}(B) \sqsubseteq V \operatorname{Vnd}\left(B^{\prime}\right)$ implies $R \operatorname{Ind}(B) \sqsubseteq R \operatorname{Ind}\left(B^{\prime}\right)$.

Proof. Assume $\operatorname{VInd}(B) \sqsubseteq \operatorname{VInd}\left(B^{\prime}\right)$. Since $\operatorname{VInd}\left(B^{\prime}\right) \in B^{\prime}$ we obtain $\operatorname{VInd}(B) \in B^{\prime}$. As by definition $R \operatorname{Ind}(B) \sqsubseteq \operatorname{VInd}(B)$, we also have $R \operatorname{Ind}(B) \in B^{\prime}$. Consequently $R \operatorname{Ind}(B)$ and $R \operatorname{Ind}\left(B^{\prime}\right)$ lie on the same branch $B^{\prime}$, and thus are comparable. For the sake of contradiction, suppose $R \operatorname{Ind}\left(B^{\prime}\right) \sqsubset R \operatorname{Ind}(B)$, then by definition of $\operatorname{VInd}$ we obtain $\operatorname{VInd}(B) \notin B^{\prime}$. Contradiction. The remaining case is the expected $\operatorname{RInd}(B) \sqsubseteq R \operatorname{Rnd}\left(B^{\prime}\right)$.

We are ready to establish the two properties wanted for VInd.

Lemma 6.11. The following holds.

(1) For every node $x,\left|\operatorname{VInd}^{-1}(x)\right|<K_{\mathrm{im}}$.

(2) If $\operatorname{VInd}\left(B^{\prime}\right) \sqsubset \operatorname{VInd}(B)$, then $\operatorname{VInd}(B) \notin B^{\prime}$.

Proof. (1): Let $B$ be an infinite branch such that $\operatorname{VInd}(B)=x$ and let $y=\operatorname{RInd}(B)$. By Lemma 6.10, important branches with the same VInd also have the same RInd and hence $\operatorname{VInd}^{-1}(x) \subseteq \operatorname{RInd}^{-1}(y)$. The desired bound follows from Lemma 6.8.

(2): By Lemma 6.10, if $\operatorname{VInd}\left(B^{\prime}\right) \sqsubset \operatorname{VInd}(B)$ then $R \operatorname{Ind}\left(B^{\prime}\right) \sqsubseteq R \operatorname{Rnd}(B)$. If $R \operatorname{Ind}\left(B^{\prime}\right) \sqsubset$ $R \operatorname{Ind}(B)$, the claim follows by definition of $\operatorname{VInd}(B)$. The case $R \operatorname{Ind}\left(B^{\prime}\right)=R \operatorname{Ind}(B)$ is not 
possible since this would imply that $\operatorname{VInd}\left(B^{\prime}\right)=\operatorname{VInd}(B)$ because they are both lying on the branch $B$ according to the assumption $\operatorname{VInd}\left(B^{\prime}\right) \sqsubset \operatorname{VInd}(B)$.

Now, for $X \in$ Atoms such that $I(X)$ is an infinite branch we define $\operatorname{BIndex}(X)$ to be $\operatorname{VInd}(I(X))$. The distribution induced by BIndex is strongly sparse:

Lemma 6.12 (strong sparsity). For $Z$ a finite zone of frontier $F,\left|B I n \operatorname{dex}^{-1}(Z)\right| \leq K_{\mathrm{im}}^{2}|F|$.

Proof. Assume that $\operatorname{VInd}(B), \operatorname{VInd}\left(B^{\prime}\right) \in Z$ for two branches $B$ and $B^{\prime}$. If the two branches exit $Z$ at the same point, i.e., if $B \cap F=B^{\prime} \cap F$, then $B$ and $B^{\prime}$ do not differ inside $Z$. As $\operatorname{VInd}(B), \operatorname{VInd}\left(B^{\prime}\right) \in Z$ we conclude that $\operatorname{VInd}(B) \in B^{\prime}$ and $\operatorname{VInd}\left(B^{\prime}\right) \in B$. Applying Lemma $6.11(2)$ yields $\operatorname{VInd}(B)=\operatorname{VInd}\left(B^{\prime}\right)$.

According to Lemmas 6.7 and 6.11 (1), the number of atoms $X$ that share the same value $B \operatorname{Index}(X)$ is bounded by $K_{\mathrm{im}}^{2}$. This shows that for each exit of the zone $Z$ there are at most $K_{\text {im }}^{2}$ branches $B$ with $\operatorname{VInd}(B) \in Z$ leaving $Z$ through that exit. Hence we obtain that $\left|\operatorname{BIndex}^{-1}(Z)\right| \leq K_{\text {im }}^{2}|F|$.

6.5. Car parking. In this subsection, our goal is to spread the indices around the tree such that each index ends in exactly one position. This can be seen as parking vehicles. In the beginning there are cars (indices) placed in the nodes of the tree, possibly more than one at the same position, and we aim at parking each of them in one node, i.e., attaching a single node to each of those cars. This is obviously not possible in general but we shall prove that, under a sparsity constraint on the distribution of vehicles, it is possible to attach a single parking place to each car, and furthermore that the mapping that, given a car, tells where to park it, can be described by a WMSO-formula.

For this purpose, we have to describe distributions and other kinds of mappings that involve integers in their domain or image by WMSO-formulas. These integers will always be bounded by some constant $K$ and hence we can split the WMSO-definition into several formulas, one for each number that may be involved. Formally, we say that a relation $R \subseteq \operatorname{dom}(t)^{r} \times I$ for some finite $I \subseteq \mathbb{Z}$ is WMSO-definable if there are WMSO-formulas $\phi_{i}\left(x_{1}, \ldots, x_{r}\right)$ for each $i \in I$ such that $\left(u_{1}, \ldots, u_{r}, i\right) \in R$ iff $t, u_{1}, \ldots, u_{r} \models \phi_{i}\left(x_{1}, \ldots, x_{r}\right)$. Note that a $K$-sparse distribution can be seen as a relation of this kind since $0 \leq D(x) \leq 3 K$ for every $x \in \operatorname{dom}(t)$.

Definition 6.13. Given a distribution $D$, a placement $P$ for $D$ is an injective partial mapping from $\operatorname{dom}(t) \times \mathbb{N}$ to $\operatorname{dom}(t)$ such that $P(x, i)$ is defined iff $i \in[D(x)]$.

A flow, defined below, can be seen as a kind of instruction on how to spread the values of a distribution to obtain a placement. In the vehicles description, this is the number of cars which will have to cross an edge in order to reach the final placement.

Recall that, for simplicity reasons, we assume that all the nodes of a tree $t$ have either 2 successors or no successors, i.e., for all nodes $u$ we have $u 0 \in \operatorname{dom}(t)$ iff $u 1 \in \operatorname{dom}(t)$. This assumption is not essential but simplifies the definitions and allows to avoid case distinctions.

Definition 6.14. A flow is a mapping $f$ from the nodes of $t$ to $\mathbb{Z}$. A flow $f$ is compatible with a distribution $D$ if for all inner nodes $x, D(x)+f(x) \leq 1+f(x 0)+f(x 1)$ and for every leaf $x, D(x)+f(x) \leq 1$. A flow $f$ is bounded by a constant $K$ if $|f(x)| \leq K$ for each node $x$. 
In this definition, $f(x)$ is interpreted as the number of cars crossing the edge from the ancestor of $x$ to $x$. In case of a negative value, $-f(x)$ cars are driving from $x$ to its ancestor. The condition of $f$ being compatible with $D$ states that after distributing all the cars according to the flow there is at most one car remaining at each node. One should note here that, according to our definition, it is possible that $f(\epsilon)<0$. With the above intuition this would mean that one has to send $-f(\epsilon)$ cars to the (non-existing) ancestor of the root. We need this case when constructing flows on finite subtrees of a given infinite tree.

In the following we show that for a $K$-sparse distribution there is a compatible flow that is bounded. From this flow we then compute a placement for the distribution. We start by defining a flow on finite trees (which can then also be used to deal with finite subtrees of a given infinite tree).

Lemma 6.15. For every finite tree $t$ and every WMSO-definable $K$-sparse distribution $D$ over $t$, there exists a WMSO-definable flow $f$ that is compatible with $D$, bounded by $2 K+1$, and such that for each node $x$ there is a zone $Z_{x}$ rooted in $x$ of frontier $F_{x}$ with

(1) $D\left(Z_{x}\right)+f(x)=\left|Z_{x}\right|+K\left(\left|F_{x}\right|-1\right)$,

(2) and $f(y) \geq K$ for all $y \in F_{x}$ different from $x$.

Proof. First note that (1) implies $f(x) \geq-K$ for each $x$ since $D$ is $K$-sparse. We define the values $f(x)$ and the zones $Z_{x}$ inductively starting at the leaves. These definitions directly imply that $f$ is compatible with $D$.

The base case of a leaf $x$ is straightforward: we set $f(x)$ to be $1-D(x)$ and $Z_{x}=\{x\}$. By the hypothesis of sparsity, we have that $D(x) \leq K+1$ and hence $|f(x)|$ is bounded by $2 K+1$.

Let $x$ be an inner node and assume that the values $f(x 0), f(x 1)$ and the zones $Z_{x 0}, Z_{x 1}$ are already defined. We set $f^{\prime}(x 0)=\min (K, f(x 0))$ and $f^{\prime}(x 1)=\min (K, f(x 1))$. Let us now define $f(x)$ to be $1+f^{\prime}(x 0)+f^{\prime}(x 1)-D(x)$ and $Z_{x}$ to contain the node $x$, the nodes of $Z_{x 0}$ if $f(x 0)<K$, and the nodes of $Z_{x 1}$ if $f(x 1)<K$. By the hypothesis of induction, we indeed obtain that $D\left(Z_{x}\right)+f(x)=\left|Z_{x}\right|+K\left(\left|F_{x}\right|-1\right)$. We illustrate this only for the case $f^{\prime}(x 0)<K$ and $f^{\prime}(x 1)=K$, the other cases are similar. In this case $Z_{x}=Z_{x 0} \cup\{x\}$, $\left|F_{x}\right|=\left|F_{x 0}\right|+1$, and $f(x)=1+f(x 0)+K-D(x)$. From this we get the following sequence of equalities:

$$
\begin{aligned}
D\left(Z_{x}\right)+f(x) & =D\left(Z_{x 0}\right)+D(x)+1+f(x 0)+K-D(x) \\
& =D\left(Z_{x 0}\right)+f(x 0)+1+K \\
& =\left|Z_{x 0}\right|+K\left(\left|F_{x 0}\right|-1\right)+1+K \\
& =\left|Z_{x 0}\right|+1+K\left|F_{x 0}\right| \\
& =\left|Z_{x}\right|+K\left(\left|F_{x}\right|-1\right) .
\end{aligned}
$$

From the definition of $f(x)$ it is clear that $f(x) \leq 2 K+1$. As mentioned before, $f(x) \geq-K$ and thus $|f(x)|$ is bounded by $2 K+1$.

It is obvious that this flow, which has only a bounded number of possible values and is defined inductively, is WMSO-definable. This definition can be done by requiring the existence of sets $X_{-K}, \ldots, X_{2 K+1}$ such that a node $x$ is in $X_{i}$ iff $f(x)=i$. This can be directly expressed if $x$ is a leaf. Otherwise it is a simple statement on the membership of the successors $x 0$ and $x 1$ of $x$. 


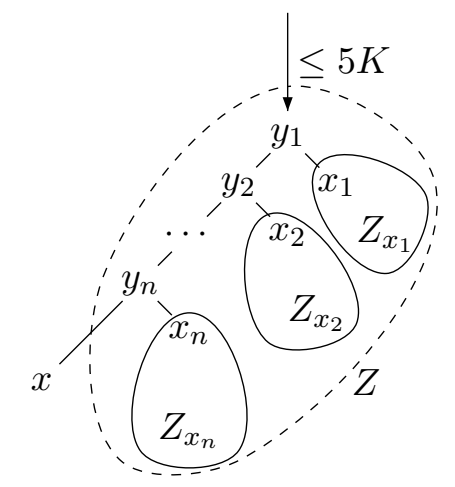

Figure 3: Proof of Lemma 6.16. The flow in nodes of type (a) is bounded by $7 K$.

Lemma 6.16. For every infinite tree $t$ and every $W M S O$-definable $K$-sparse distribution $D$, there exists a WMSO-definable flow $f$ bounded by $7 K$ that is compatible with $D$ such that $f(\epsilon)=0$.

Proof. As a $K$-sparse distribution restricted to a finite subtree of $t$ is also $K$-sparse on this subtree, we can apply Lemma 6.15 to define the values of $f(x)$ for the nodes $x$ that are not on infinite branches of $t$.

Let $B$ be the set of nodes appearing in some infinite branch. We define inductively for any node $x \in B$ a flow $f(x)$ such that $0 \leq f(x) \leq 7 K$. We only consider non-negative values since on infinite branches we never reach a leaf and hence there is no need for an upward flow.

We start by setting $f(\epsilon)=0$. For $x \neq \epsilon$ let $y \in B$ be the father of $x$. Three cases may happen. If at node $y$ only one of its children is in $B$ (case (a)), we forward everything to this node. Otherwise (cases (b) and (c)), we forward at most $5 K$ to the left child and the rest to the right child. The formal definitions are given below, where the the max operator is only used to avoid negative flows.

(a) If $x$ is the only child of $y$ in $B$, then we set

$$
f(x)=\max \left(0, f(y)+D(y)-f\left(x^{\prime}\right)-1\right)
$$

where $x^{\prime}$ is the other child of $y$.

(b) If the two children of $y$ are in $B$ and $x$ is the left child, then we set

$$
f(x)=\max (0, \min (5 K, f(y)+D(y)-1)) .
$$

(c) If the two children of $y$ are in $B$ and $x$ is the right child, then we set

$$
f(x)=\max (0, f(y)+D(y)-1-5 K) .
$$

Obviously, $f(x) \geq 0$ in all cases. We show that if $x$ is of type (b) or (c), then $f(x) \leq 5 K$, and if $x$ is of type (a), then $f(x) \leq 7 K$.

In case (b), $f(x) \leq 5 K$ follows directly from the definition and in case (c) from $f(y) \leq$ $7 K$ (by induction) and $D(y) \leq 3 K+1$ ( $D$ is $K$-sparse).

For $x$ as in (a) we cannot use a local argument but we have to go upwards until we reach a node that has a flow of at most $5 K$. Such a node must exist because we eventually meet a node of type (b) or (c), or the root, which has flow 0. All nodes we met before must be of type (a). 
The following definitions are illustrated in Figure 3. The choice of the $y_{i}$ being the left successors in the figure is arbitrary and only for matters of presentation. Let $y_{n}, \ldots, y_{1}$ be such that $y_{n}$ is the father of $x, y_{i-1}$ is the father of $y_{i}$ for all $i \in\{2, \ldots, n\}, f\left(y_{1}\right) \leq 5 K$, and $f\left(y_{2}\right), \ldots, f\left(y_{n}\right)>5 K$. As mentioned above, $y_{2}, \ldots, y_{n}$ are of type (a).

Let $x_{1}, \ldots, x_{n}$ be such that $y_{i}$ is the father of $x_{i}, x_{n} \neq x$, and $x_{i} \neq y_{i+1}$ for $i \in$ $\{1, \ldots, n-1\}$, i.e., $x_{i}$ is the brother of $y_{i+1}$. For the $x_{i}$ the flow is defined using Lemma 6.15 because they are not lying on an infinite branch. Hence there are zones $Z_{x_{i}}$ rooted at $x_{i}$ of frontier $F_{x_{i}}$ such that

$$
D\left(Z_{x_{i}}\right)+f\left(x_{i}\right)=\left|Z_{x_{i}}\right|+K\left(\left|F_{x_{i}}\right|-1\right) .
$$

Let $Z=\bigcup_{i=1}^{n}\left(\left\{y_{i}\right\} \cup Z_{x_{i}}\right)$ and let $F$ be the frontier of $Z$. Then

$$
\begin{aligned}
|Z| & =n+\sum_{i=1}^{n}\left|Z_{x_{i}}\right| \\
|F| & =2+\sum_{i=1}^{n}\left(\left|F_{x_{i}}\right|-1\right) \\
\sum_{i=1}^{n} D\left(y_{i}\right) & =D(Z)-\sum_{i=1}^{n} D\left(Z_{x_{i}}\right)
\end{aligned}
$$

Since $y_{2}, \ldots, y_{n}$ are of type (a) and furthermore their flow is bigger than $5 K$ (and hence bigger than 0 ), we get

$$
f(x)=f\left(y_{1}\right)+\sum_{i=1}^{n}\left(D\left(y_{i}\right)-1-f\left(x_{i}\right)\right) .
$$

We know that $f\left(y_{1}\right) \leq 5 K$ and hence it remains to be shown that $\sum_{i=1}^{n}\left(D\left(y_{i}\right)-1-f\left(x_{i}\right)\right) \leq$ $2 K$. This can be deduced as follows:

$$
\begin{aligned}
& \sum_{i=1}^{n}\left(D\left(y_{i}\right)-1-f\left(x_{i}\right)\right) \stackrel{\text { 6.4 }}{=} D(Z)-n-\sum_{i=1}^{n}\left(D\left(Z_{x_{i}}\right)+f\left(x_{i}\right)\right) \\
& \stackrel{6.1}{=} D(Z)-n-\sum_{i=1}^{n}\left(\left|Z_{x_{i}}\right|+K\left(\left|F_{x_{i}}\right|-1\right)\right) \\
& \stackrel{6.3}{=} D(Z)-n-K(|F|-2)-\sum_{i=1}^{n}\left|Z_{x_{i}}\right| \\
& \stackrel{K \text {-sparse }}{\leq}|Z|+K|F|-n-K(|F|-2)-\sum_{i=1}^{n}\left|Z_{x_{i}}\right| \\
& \text { 6.2 } 2 K
\end{aligned}
$$

That this flow $f$ is compatible with $D$ and that it is WMSO-definable can easily be deduced from the definitions.

We are now ready to establish our placement Lemma.

Lemma 6.17 (car parking). For every tree $t$ and every WMSO-definable $K$-sparse distribution $D$, there exists a WMSO-definable placement for $D$. If $t$ is finite, we additionally require that $D(\operatorname{dom}(t)) \leq|\operatorname{dom}(t)|$. 
Proof. According to Lemmas 6.15 and 6.16 we know that there is a WMSO-definable flow $f$ that is compatible with $D$. For simplicity, we first assume that $f(\epsilon)=0$, which is always the case for infinite trees (Lemma 6.16). If $f(\epsilon)>0$ for finite trees, then we can simply redefine $f(\epsilon)=0$ without changing the property of $f$ being compatible with $D$. If $t$ is finite and $f(\epsilon)<0$, then we cannot simply set $f(\varepsilon)=0$ because this would affect the compatibility of $f$ with $D$. At the end of the proof we briefly explain how to treat this case.

The general strategy for defining the placement is the following:

- From each node we send all the cars except one to its neighbors. The number of cars sent to each neighbor is described by the flow.

- If we follow this strategy, then each edge in $t$ is crossed by the cars in only one direction. Hence, a car cannot visit the same node twice. This means that it might be sent up in the tree for some steps and from some point onwards it is only sent downwards.

- To be sure that each car will be parked after finite time we order all the cars that cross a node (as described by $D$ and $f$ ) according to a fixed strategy and we also fix a scheme for distributing the cars to the neighboring nodes.

- This ordering will ensure that the index of a car decreases each time it is sent down in the tree. As described above, each car is sent up in the tree only a finite number of times. Hence, if we always park the car that is first in the ordering at a specific node, then each car will eventually be parked.

To show that this strategy can be realized by WMSO-formulas we first describe the ordering of cars that we use and then define formulas

- $\operatorname{send}_{i}(x, y)$ meaning that the $i$ th car at node $x$ is sent to node $y$.

- drive $_{i, j}(x, y)$ meaning that the $i$ th car at node $x$ is sent to $y$ and is car number $j$ at $y$.

- $\operatorname{start}_{i}(x)$ meaning that the $i$ th car at node $x$ does not come from another node.

- itinerary $_{i}\left(x, X_{1}, \ldots, X_{K}, y\right)$ meaning that the $i$ th car at node $x$ will be parked at node $y$ using an itinerary that is described by the sets $X_{1}, \ldots, X_{K}$.

To define these formulas we first have to introduce some notation. To avoid case distinctions we define $f^{+}(x)=\max (f(x), 0)$ and $f^{-}(x)=\min (f(x), 0)$. Furthermore, we assume by convention that for a leaf $x$ the values $f^{+}(x 0), f^{+}(x 1), f^{-}(x 0), f^{-}(x 1)$ are defined, and are all set to 0 .

Then the number of cars crossing a node $x$ is $f^{+}(x)+f^{-}(x 0)+f^{-}(x 1)+D(x)$. Since all the values involved in this expression are bounded and since we can increase $K$ without affecting the $K$-sparsity of $D$, we can assume that $f^{+}(x)+f^{-}(x 0)+f^{-}(x 1)+D(x) \leq K$ for all $x$.

Since $f$ is WMSO-definable, we can also assume that there are formulas $\phi_{i}^{+}(x)$ and $\phi_{i}^{-}(x)$ defining $f^{+}$and $f^{-}$. Then expressions of the form $i_{1}+f^{+}(x)+f^{-}(y)=i_{2}$ for $i_{1}, i_{2} \in[K]$ can easily be expressed as Boolean combinations of the formulas $\phi_{i}^{+}$and $\phi_{i}^{-}$. The use of expressions of this kind simplifies the presentation of the formulas.

We start by giving the orderings used in the definitions of the formulas $\operatorname{send}_{i}$ and drive $_{i, j}$. The cars that cross a node $x$ will be distributed in the following order that we refer to as the distribution order:

$$
\begin{array}{|l|l|l|l|}
\hline f^{+}(x) & f^{-}(x 0) & f^{-}(x 1) & D(x) \\
\hline
\end{array}
$$


That is, we first distribute the cars that come from the father of $x$, then the cars that come from the left child of $x$, and so on. It remains to fix where to send the cars.

\begin{tabular}{|l|l|l|l|}
\hline 1 & $f^{-}(x)$ & $f^{+}(x 0)$ & $f^{+}(x 1)$ \\
\hline
\end{tabular}

This means that the first car in the distribution order is parked in the node $x$. The next cars are sent to the father of $x$ if $f(x)$ is negative. The following cars in the distribution order are send to the left child of $x$ and the remaining cars to the right child of $x$.

To illustrate this, consider the following example with $x=y 0, f^{+}(y)=3, f^{-}(x)=5$, and $f^{+}(y 1)=7$.

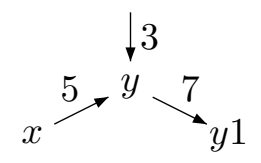

Let us first see how the cars at $y$ are ordered according to the distribution order. The first three are the ones coming from the father of $y$. The next 5 are those coming from $x$. There are no cars coming from $y 1$, and the last cars are those from $D(y)$. Now, we would like to know what happens to the 4 th car at $x$. The first car at $x$ stays at $x$. The next 5 cars are sent to $y$, that is, the fourth car at $x$ is the third car sent from $x$ to $y$. According to the distribution order at $y$ described before, this car becomes car number 6 at $y$.

This is expressed by the following formulas, where the first two are only defined for $2 \leq i \leq K$ because the first car is always kept at the current position.

- For $2 \leq i \leq K$ :

$$
\begin{aligned}
\operatorname{send}_{i}(x, y):= & {\left[(x=y 0 \vee x=y 1) \wedge 1<i \leq C_{1}\right] } \\
& \vee\left[x 0=y \wedge C_{1}<i \leq C_{2}\right] \\
& \vee\left[x 1=y \wedge C_{2}<i \leq C_{3}\right]
\end{aligned}
$$

Here $C_{1}=1+f^{-}(x), C_{2}=1+f^{-}(x)+f^{+}(x 0)$, and $C_{3}=1+f^{-}(x)+f^{+}(x 0)+$ $f^{+}(x 1)$.

- For $2 \leq i \leq K$ :

$$
\begin{aligned}
\operatorname{drive}_{i, j}(x, y):= & \operatorname{send}_{i}(x, y) \wedge \\
& \left(\left[x=y 0 \wedge j=i-1+f^{+}(y)\right]\right. \\
& \vee\left[x=y 1 \wedge j=i-1+f^{+}(y)+f^{-}(y 0)\right] \\
& \vee\left[x 0=y \wedge j=i-1-f^{-}(x)\right] \\
& \left.\vee\left[x 1=y \wedge j=i-1-f^{-}(x)-f^{+}(x 0)\right]\right)
\end{aligned}
$$

- For $1 \leq i \leq K$ :

$$
\operatorname{start}_{i}(x):=f^{+}(x)+f^{-}(x 0)+f^{-}(x 1)<i \leq f^{+}(x)+f^{-}(x 0)+f^{-}(x 1)+D(x)
$$

- For $1 \leq i \leq K$ :

$$
\begin{aligned}
\operatorname{itinerary}_{i}\left(x, X_{1}, \ldots, X_{K}, y\right):= & \operatorname{disjoint}\left(\mathrm{X}_{1}, \ldots, \mathrm{X}_{\mathrm{K}}\right) \wedge \\
& x \in X_{i} \wedge \operatorname{start}_{i}(x) \wedge X_{1}=\{y\} \\
& \wedge \bigwedge_{j=2}^{K}\left(\forall z \in X_{j} \underset{j^{\prime} \in[K]}{\bigvee} \exists z^{\prime} \in X_{j^{\prime}}: \operatorname{drive}_{j, j^{\prime}}\left(z, z^{\prime}\right)\right)
\end{aligned}
$$

This formula states that the $i$ th car at $x$ starts there. The free set variables describe the set of positions that this car crosses, where a position is included in $X_{m}$ if the 
car is the $m$ th one at this position. Finally, it states that $y$ is the only position where the car is first in the ordering. Hence it will stop at $y$.

Then the formulas $\psi_{i}(x, y)$ defining the placement are given by

$$
\psi_{i}(x, y)=\exists X_{1}, \ldots X_{K}\left(\operatorname{itinerary}_{i}\left(x, X_{1}, \ldots, X_{K}, y\right)\right) .
$$

As mentioned at the beginning of the proof we now discuss how to treat the case $f(\epsilon)<0$. Recall that this may only happen for finite trees. In general, simply redefining $f(\epsilon)=0$ may lead to a flow that is not compatible with $D$ anymore. Therefore, we have to use a different strategy.

The strategy for distributing the cars described above would lead to $f^{-}(\epsilon)$ cars that "get stuck" at the root because, following the flow, they should be sent upwards, and this is not possible. This means that there are at most $K$ cars (for simplicity assume exactly $K$ cars) that start at some node but never arrive at some destination, i.e., there are nodes $x_{1}, \ldots, x_{K}$ and $i_{1}, \ldots, i_{K} \in[K]$ such that

$$
\operatorname{start}_{i_{j}}\left(x_{j}\right) \wedge \neg \exists y\left(\psi_{i_{j}}\left(x_{j}, y\right)\right)
$$

is satisfied. From the assumption $D(\operatorname{dom}(t)) \leq|\operatorname{dom}(t)|$ we can conclude that there remain at least $K$ nodes where no car is parked, i.e., $K$ nodes which are not image of the function defined by the $\psi_{i}$ 's. Let $y_{1}, \ldots, y_{K}$ be the $K$ first such nodes for some WMSO-definable order (this is possible since the tree is finite). We can now extend this function by ordering the $x_{1}, \ldots, x_{K}$ and map the $i_{j}$ th car from $x_{j}$ to $y_{j}$. Note that these definitions are expressible in WMSO. In this way, we obtain a modification of the function defined by the $\psi_{i}$ 's into a placement for $D$.

6.6. Proof of Theorem 4.3. We can now prove Theorem 4.3 as stated in Section 4 by combining the previous results.

For $X \in$ Atoms let

$$
\operatorname{Index}(X)=\left\{\begin{array}{l}
\operatorname{BIndex}(X) \text { if } I(X) \text { is an infinite branch, } \\
\operatorname{SIndex}(X) \text { otherwise. }
\end{array}\right.
$$

We construct a formula $\phi_{\text {ind }}(X, y)$ that associates to each $X \in$ Atoms its index $y=$ $\operatorname{Index}(X)$. From the definitions of $I(X), \operatorname{SIndex}(X)$, and $B \operatorname{Index}(X)$ it is clear that we can construct such a formula. Note that in the definition of this formula, we do not have to explicitly represent infinite sets (though $I(X)$ may be infinite) because we can construct a WMSO formula $\phi_{i m p}(X, x)$ that associates to $X \in$ Atoms its important nodes. From this one can construct WMSO definitions of $\operatorname{SIndex}(X)$ and $B \operatorname{Index}(X)$, and hence the formula $\phi_{\text {ind }}(X, y)$ is also WMSO.

Then, we compute the distribution $D$ defined by $D(x)=\left|\operatorname{Index}^{-1}(x)\right|$. Since this distribution is $\left(K_{\mathrm{s}}+K_{\mathrm{im}}^{2}\right)$-sparse by Lemmas 6.6 and 6.12, it is also WMSO-definable using the formula $\phi_{\text {ind }}$. Let $K$ be a constant such that $D(x) \leq K$ for all nodes $x$. Applying Lemma 6.17, we obtain a WMSO-definable placement $P$ for $D$. One should note that for finite $t$ the assumption $D(\operatorname{dom}(t)) \leq|\operatorname{dom}(t)|$ is satisfied because $D(\operatorname{dom}(t))$ is the number of elements in the set $E$, and $\mathcal{I}(t)$ being isomorphic to $\mathcal{P}^{F}(E)$ implies that $t$ has at least as many elements as $E$.

Let $\psi_{i}\left(x_{1}, x_{2}\right)$ for $i \in[K]$ be the formulas defining $P$. Now, we order all the $X \in$ Atoms with the same index. A possible definition for such an ordering is $X<Y$ if the lexicographically smallest node that is not in $X \cap Y$ is in $X$. Then one can construct 
WMSO-formulas $\theta_{i}(X)$ stating that $X$ is the $i$ th set in the ordering among those that have the same index as $X$.

The WMSO-formula $\operatorname{Code}(X, x)$ that attaches $X$ to its final position $x$ is then defined as follows:

$$
\operatorname{Code}(X, x)=\bigvee_{i \in[K]}\left(\theta_{i}(X) \wedge \exists y\left(\phi_{\text {ind }}(X, y) \wedge \psi_{i}(y, x)\right)\right.
$$

One should note here that without the results from Subsection 6.5 we can obtain a weaker version of Corollary 4.4 by replacing the interpretation $\mathcal{I}_{2}$ by a transduction, i.e., an interpretation that can use a fixed number $K$ of copies of the given structure. Such a transduction can be realized using the formula $\phi_{\text {ind }}$ instead of Code. In particular one could use this weaker version of the result in all the applications, but at the price of some notational and technical overheads.

From this point of view, Lemma 6.17 can also be seen as a result on the question under which conditions a (W)MSO-transduction is equivalent to a (W)MSO-interpretation on binary trees. Namely, if the distribution defined by the transduction, i.e., the function assigning to each node the number of times it is used in the result of the transduction, is $K$-sparse (for some $K$ ) on each tree. And this presentation can be used either for WMSOtransductions or MSO-transductions.

Acknowledgments. We are particularly grateful to Vince Bárány for his contribution to the random-graph proof, as well as his comments on previous versions of this work. We also thank Achim Blumensath for commenting on earlier stages of this work, and the anonymous referees for their helpful comments.

\section{REFERENCES}

[Bár06] Vince Bárány. Invariants of automatic presentations and semi-synchronous transductions. In STACS 2006, volume 3884 of LNCS, pages 289-300. Springer, 2006.

[BG00] Achim Blumensath and Erich Grädel. Automatic Structures. In Proceedings of 15th IEEE Symposium on Logic in Computer Science LICS 2000, pages 51-62, 2000.

[BG04] Achim Blumensath and Erich Grädel. Finite presentations of infinite structures: Automata and interpretations. Theory of Computing Systems, 37:641 - 674, 2004.

[Blu99] Achim Blumensath. Automatic structures. Diploma thesis, RWTH-Aachen, 1999.

[Blu01] Achim Blumensath. Prefix-recognisable graphs and monadic second-order logic. Technical Report AIB-06-2001, RWTH Aachen, May 2001.

[Cau96] Didier Caucal. On infinite transition graphs having a decidable monadic theory. In $I C A L P$ '96, volume 1099 of LNCS, pages 194-205. Springer, 1996.

[Cau02] Didier Caucal. On infinite terms having a decidable monadic theory. In MFCS'02, volume 2420 of $L N C S$, pages 165-176. Springer, 2002.

[CO06] Bruno Courcelle and Sang-il Oum. Vertex-minors, monadic second-order logic and a conjecture by seese. Journal of Combinatorial Theory, Series B, 2006. to appear.

[Col02] Thomas Colcombet. On families of graphs having a decidable first order theory with reachability. In Proceedings of ICALP 2002, volume 2380 of LNCS, pages 98-109. Springer, 2002.

[Col04] Thomas Colcombet. Equational presentations of tree-automatic structures. In Workshop on Automata, Structures and Logic, Auckland, New Zealand, December 2004.

[Cou89] Bruno Courcelle. The monadic second order logic of graphs II: Infinite graphs of bounded width. Mathematical System Theory, 21:187-222, 1989. 
[Cou97] Bruno Courcelle. The expression of graph properties and graph transformations in monadic second-order logic. In G. Rozenberg, editor, Handbook of graph grammars and computing by graph transformations, Vol. 1 : Foundations, chapter 5, pages 313-400. World Scientific, 1997.

[Cou04] Bruno Courcelle. Clique-width of countable graphs: a compactness property. Discrete Mathematics, 276(1-3):127-148, 2004.

[CT02] Olivier Carton and Wolfgang Thomas. The monadic theory of morphic infinite words and generalizations. Information and Computation, 176(1):51-65, 2002.

[CW03] Arnaud Carayol and Stefan Wöhrle. The Caucal hierarchy of infinite graphs in terms of logic and higher-order pushdown automata. In FSTTCS'03, volume 2914 of LNCS, pages 112-123. Springer, 2003.

[DT90] Max Dauchet and Sophie Tison. The theory of ground rewrite systems is decidable. In LICS'90, pages 242-248. IEEE, 1990.

$\left[\mathrm{ECH}^{+}\right.$92] David B. A. Epstein, James W. Cannon, Derek F. Holt, Silvio V. F. Levy, Michael S. Paterson, and William P. Thurston. Word processing in groups. Jones and Barlett publishers, 1992.

[ER66] Calvin C. Elgot and Michael O. Rabin. Decidability and undecidability of extensions of second (first) order theory of (generalized) successor. Journal of Symbolic Logic, 31(2):169-181, 1966.

[Hod83] Bernard R. Hodgson. Décidabilité par automate fini. Ann. Sci. Math. Québec, 7(3):39-57, 1983.

[Hod93] Wilfrid Hodges. Model Theory. Cambridge University Press, 1993.

[KL02] Dietrich Kuske and Markus Lohrey. On the theory of one-step rewriting in trace monoids. In ICALP'02, volume 2380 of $L N C S$, pages 752-763. Springer, 2002.

[KN95] Bakhadyr Khoussainov and Anil Nerode. Automatic presentations of structures. In Workshop $L C C$ '94, volume 960 of $L N C S$, pages 367-392. Springer, 1995.

[KNRS04] Bakhadyr Khoussainov, André Nies, Sasha Rubin, and Frank Stephan. Automatic structures:

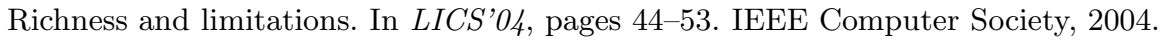

[KNUW05] Teodor Knapik, Damian Niwiński, Paweł Urzyczyn, and Igor Walukiewicz. Unsafe grammars, panic automata, and decidability. In ICALP'05, volume 3580 of $L N C S$, pages 1450-1461. Springer, 2005.

[KRS04] Bakhadyr Khoussainov, Sasha Rubin, and Frank Stephan. Definability and regularity in automatic structures. In $S T A C S^{\prime} 04$, volume 2996 of $L N C S$, pages 440-451. Springer, 2004.

[Mad03] P. Madhusudan. Model-checking trace event structures. In LICS'03, pages 371-380. IEEE Computer Society, 2003.

[MR99] Johann A. Makowsky and Udi Rotics. On the clique-width of graphs with few $\mathrm{p}_{4}$ 's. International Journal of Foundations of Computer Science, 10(3):329-348, 1999.

[MS85] David E. Muller and Paul E. Schupp. The theory of ends, pushdown automata, and second-order logic. Theoretical Computer Science, 37:51-75, 1985.

[Rub04] Sasha Rubin. Automatic Structures. PhD thesis, University of Auckland, New Zealand, 2004.

[See91] Detlef Seese. The structure of models of decidable monadic theories of graphs. Annals of Pure and Applied Logic, 53:169-195, 1991.

[Tho97] Wolfgang Thomas. Languages, automata, and logic. In G. Rozenberg and A. Salomaa, editors, Handbook of Formal Language Theory, volume III, pages 389-455. Springer, 1997.

[Tho03] Wolfgang Thomas. Constructing infinite graphs with a decidable mso-theory. In Proceedings of MFCS 2003, volume 2747 of LNCS, pages 113-124. Springer, 2003. 